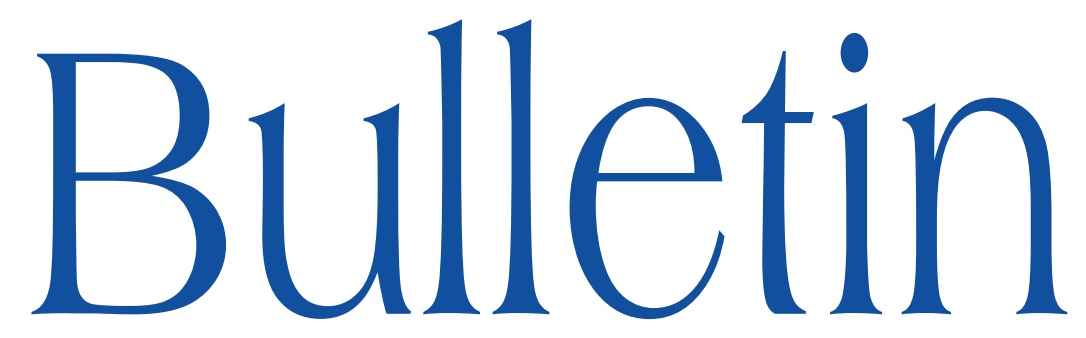

de la SOCIÉTÉ MATHÉMATIQUE DE FRANCE

\title{
PROPRIÉTÉS DE MÉLANGE DU FLOT DES CHAMBRES DE WEYL DES GROUPES DE PING-PONG
}

\author{
Xavier Thirion
}

Tome 137

Fascicule 3

2000 


\title{
PROPRIÉTÉS DE MÉLANGE DU FLOT DES CHAMBRES DE WEYL DES GROUPES DE PING-PONG
}

\author{
PAR XAVIER THIRION
}

\begin{abstract}
Résumé. - Dans cet article, nous étudions le flot des chambres de Weyl d'une large classe de sous-groupe discrets d'un groupe de Lie semi-simple réel : les groupes de Ping-Pong. Nous montrons que ce flot est mélangeant relativement à la mesure de Patterson-Sullivan ; celle-ci étant infinie en rang $\geq 2$, nous précisons cette propriété de mélange en explicitant sa vitesse dans le direction du vecteur de croissance du groupe.

Abstract (Mixing properties of Weyl Chamber flows of Ping-Pong groups)

In this paper, we study the Weyl's chamber flow associated with a large class of discrete subgroups of a semi-simple real Lie group : the Ping-Pong groups. We prove that this flow is mixing with respect to the Patterson-Sullivan measure ; this measure being infinite in rank $\geq 2$, we precise this mixing property with a precise speed of convergence in the direction of the growth vector of the group.
\end{abstract}

\section{Introduction}

Soit $G$ un groupe de Lie linéaire réel, connexe et semi-simple de rang $r g(G)$ dont l'espace symétrique associé $\left(X, d_{X}\right)$ est de type non compact.

Dans le cas où $G$ désigne le groupe orthochrone $S O_{o}(1, n)$, l'espace symétrique associé est l'espace hyperbolique $\mathbb{H}^{n}$ et les groupes de Ping-Pong ont été

Texte reçu le 18 janvier 2009, révisé le 21 octobre 2008, accepté le 4 décembre 2008

Xavier Thirion, Département de Mathématiques, Institut Galilée, Université Paris 13, 99, avenue Jean-Baptiste Clément, 93430 - Villetaneuse

- E-mail : xthirion@math.univparis13.fr

Classification mathématique par sujets (2000). - 22E40; 53C35.

Mots clefs. - Lie groups, discrete subgroups, higher rank. 
introduits et étudiés par F. Dal'bo et M. Peigné [7]. Dans le cadre plus large où nous nous plaçons, ils représentent une généralisation des groupes de Schottky utilisés par Y. Benoist [5] et fournissent une classe maniable de sous-groupes discrets et Zariski denses de $G$. Par exemple si $G$ désigne $S O_{o}(1, n)$ et $\Gamma$ un groupe de Ping-Pong au sens de [7], le flot géodésique de $\Gamma \backslash \mathbb{H}^{n}$ possède un codage explicite.

Dans cet article, nous étendons la définition de groupe de Ping-Pong et nous étudions les propriétés des mesures de Patterson-Sullivan associées suivant la généralisation aux espaces symétriques de rang supérieur proposée par $\mathrm{P}$. Albuquerque [1].

Afin d'énoncer les principaux résultats de ce travail, introduisons quelques notations. Soit $\mathfrak{a}$ une sous-algèbre de Cartan de l'algèbre de Lie $\mathfrak{g}$ de $G$ et soit $K$ un sous-groupe compact maximal. On choisit une chambre de Weyl ouverte $\mathfrak{a}^{++}$et on note $\mathfrak{a}^{+}$l'adhérence de $\mathfrak{a}^{++}$dans $\mathfrak{a}$. On dispose alors de la décomposition $G=K \exp \left(\mathfrak{a}^{+}\right) K$ et de la projection de Cartan $\mu: G \rightarrow \mathfrak{a}^{+}$ associée. On munit $\mathfrak{a}$ de la norme euclidienne $\|$.$\| telle que, pour tout g \in G$, on a $d_{X}(o, g \cdot o)=\|\mu(g)\|$ et on note $\langle.,$.$\rangle le produit scalaire associé à \|$.$\| .$

On fixe un groupe de Ping-Pong $\Gamma$ (nous renvoyons le lecteur à la section 6.1 pour une description précise de ces groupes) et on note $\left(\mathcal{W}_{\Gamma},\left(w_{\Gamma}^{\alpha} / \alpha \in \mathfrak{a}\right)\right)$ le flot des chambres de Weyl de $\Gamma$.

Nous nous intéressons ensuite aux propriétés ergodiques des mesures de Patterson-Sullivan. Pour ce faire, nous renvoyons le lecteur aux sections 4 et 7.0.0.1 où nous rappelons la définition du vecteur de croissance et la construction des mesures de Patterson-Sullivan.

Nous avons le

THÉORÈme 1.1. - Il existe une mesure de Patterson-Sullivan $m_{\Gamma}$ sur $\mathcal{W}_{\Gamma}$ telle que, pour toutes fonctions $\psi_{1}, \psi_{2}: \mathcal{W}_{\Gamma} \rightarrow \mathbb{R}$ continues à support compact, la fonction

$$
\mathfrak{a} \rightarrow \mathbb{R}, \quad \alpha \mapsto\langle\alpha, \tau(\Gamma)\rangle^{\frac{r g(X)-1}{2}} m_{\Gamma}\left(\psi_{1} \circ w_{\Gamma}^{\alpha} \times \psi_{2}\right)
$$

soit bornée. De plus, il existe $c>0$ et un endomorphisme $S$ sur a symétrique et défini positif tels que, pour tout $\alpha \in \mathfrak{a}$ et toutes fonctions $\psi_{1}, \psi_{2}: \mathcal{W}_{\Gamma} \rightarrow \mathbb{R}$ continues à support compact, on ait

$t^{\frac{r g(X)-1}{2}} m_{\Gamma}\left(\psi_{1} \circ w_{\Gamma}^{t \tau(\Gamma)+\sqrt{t} \alpha} \times \psi_{2}\right) \rightarrow c e^{-\frac{\|\alpha\|^{2}\|\tau(\Gamma)\|^{2}-\langle\alpha, \tau(\Gamma)\rangle^{2}}{\|\tau(\Gamma)\|^{2}}} m_{\Gamma}\left(\psi_{1}\right) m_{\Gamma}\left(\psi_{2}\right)$ lorsque $t \rightarrow+\infty$.

Lorsque le rang géométrique de $X$ vaut 1 , le flot des chambres de Weyl s'identifie avec le flot géodésique de $\Gamma \backslash X$. Ainsi, dans ce contexte, la mesure 
de Patterson-Sullivan est finie et le dernier résultat exprime le fait que le flot géodésique est mélangeant par rapport à la mesure de Patterson-Sullivan.

La propriété importante des groupes de Ping-Pong que nous utilisons ici est que leur ensemble limite peut être codé à l'aide d'un alphabet dénombrable. La restriction du flot des chambres de Weyl à un borélien « convenable » est alors semi-conjuguée à un flot spécial au dessus d'une transformation dilatante possédant une infinité dénombrable de branches inverses. Ce qui nous permet d'adapter notre étude aux travaux de Y. Guivarc'h et J. Hardy [10] en utilisant le théorème du renouvellement pour les chaînes semi-markoviennes dû à $M$. Babillot [2].

Pour l'élaboration de ce travail, j'ai bénéficié des remarques et suggestions de Marc Peigné. Je tiens ici à l'en remercier.

\section{Notations}

Dans cette section, nous fixons quelques notations que nous utiliserons tout au long de ce texte.

Notations relatives à la structure de groupe de Lie de $G$. - On note $(\mathfrak{g},[.,]$. l'algèbre de Lie de $G$, Ad la représentation adjointe de $G$ sur $\mathfrak{g}$ et exp l'application exponentielle de $\mathfrak{g}$ sur $G$.

Involution de Cartan et décomposition de Cartan. - Soit $\Theta$ une involution de Cartan de $\mathfrak{g}$ et $\mathfrak{g}=\mathfrak{k} \oplus \mathfrak{p}$ la décomposition de Cartan associée : $\mathfrak{k}=\{X \in$ $\mathfrak{g} / \Theta(X)=X\}$ et $\mathfrak{p}=\{X \in \mathfrak{g} / \Theta(X)=-X\}$.On fixe un sous-espace abélien maximal $\mathfrak{a}$ de $\mathfrak{p}$. On note $A$ le sous-groupe de $G$ dont $\mathfrak{a}$ est l'algèbre de Lie.

Racines, chambres de Weyl. - On note $R$ l'ensemble des racines de a dans $\mathfrak{g}$; c'est-à-dire, l'ensemble des formes linéaires non nulles $\chi$ sur a tel que le sous-espace

$$
\mathfrak{g}_{\chi}:=\{X \in \mathfrak{g} / \forall Y \in \mathfrak{a}, \quad[X, Y]=\chi(Y) X\}
$$

soit non nul. Chacune des racines de $\mathfrak{a}$ s'annule sur un hyperplan de a. On appelle chambres de Weyl de a les composantes connexes de a privé de ces hyperplans. On fixe une de ces chambres de Weyl notée $\mathfrak{a}^{++}$. On note $\mathfrak{a}^{+}$ l'adhérence de $\mathfrak{a}^{++}$dans $\mathfrak{a}$.

Sous-groupe compact maximal. - On note $K$ le sous-groupe de $G$ dont $\mathfrak{k}$ est l'algèbre de Lie. C'est un sous-groupe compact maximal de $G$.

Composante de Cartan. - Pour tout $g \in G$, il existe un unique élément $\mu(g) \in \mathfrak{a}^{+}$, appelé composante de Cartan de $g$, tel que $\exp (\mu(g)) \in K g K$. L'application $\mu: G \rightarrow \mathfrak{a}^{+}$ainsi définie est appelée projection de Cartan. 
Norme euclidienne sur $\mathfrak{a}$. - La projection de Cartan permet de munir a d'une norme euclidienne $\|$.$\| provenant de la métrique riemannienne de X$ telle que, pour tout $g \in G$, on ait $d_{X}(o, g \cdot o)=\|\mu(g)\|$.

Involution d'opposition. - On note $\iota$ l'unique involution, dite d'opposition, de $\mathfrak{a}$ telle que, pour tout $g \in G$, on ait $\iota(\mu(g))=\mu\left(g^{-1}\right)$.

Racines positives et simples. - Une racine $\chi \in R$ est dite positive si elle est positive sur $\mathfrak{a}^{+}$. On note $R^{+}$l'ensemble des racines positives et on pose

$$
\mathfrak{n}:=\sum_{\chi \in R^{+}} \mathfrak{g}_{\chi}
$$

On note $N$ le sous-groupe connexe de $G$ dont $\mathfrak{n}$ est l'algèbre de Lie. Une racine positive est dite simple si elle ne peut s'écrire comme la somme de deux racines positives. On note $\Delta$ l'ensemble des racines simples.

Décomposition d'Iwasawa. - Pour tout $g \in G$, il existe un unique $(k, \alpha, n) \in$ $K \times \mathfrak{a} \times N$ tel que $g=k \exp (\alpha) n$. De plus, l'application

$$
K \times \mathfrak{a} \times N \rightarrow G \quad(k, \alpha, n) \mapsto k \exp (\alpha) n
$$

est un difféomorphisme de classe $C^{\infty}$, appelé décomposition d'Iwasawa.

Centralisateur de $A$ dans $K$ et sous groupe parabolique minimal. - On note $M$ le centralisateur de $A$ dans $K$ et on pose $P=M A N$. Le groupe $M A$ normalise $N$ donc $P$ est un sous-groupe de $G$.

Composante de Jordan. - Tout élément $g \in G$ admet une décomposition unique, appelée décomposition de Jordan, $g=g_{e} g_{h} g_{u}$ en produit de trois éléments qui commutent, avec $g_{e}$ elliptique (c'est-à-dire semi-simple et contenu dans un sous-groupe compact de $G$ ), $g_{h}$ hyperbolique (c'est-à-dire conjugué à un élément $\exp (a(g))$ avec $\left.a(g) \in \mathfrak{a}^{+}\right)$et $g_{u}$ unipotent. On note $\lambda(g)=a(g)$.

Exemples. - (1) Explicitons certains de ces objets dans le cas où $G$ désigne le groupe orthochrone $S O_{o}(1, n)$, c'est-à-dire la composante connexe de l'identité dans le sous-groupe de $G L(n+1, \mathbb{R})$ formé des matrices $g \in G L(n+1, \mathbb{R})$ telles que $g I_{1, n}{ }^{t} g=I_{1, n}$, où

$$
I_{1, n}=\left(\begin{array}{cccc}
1 & & & (0) \\
& -1 & & \\
& & \ddots & \\
(0) & & & -1
\end{array}\right) .
$$

L'algèbre de Lie $\mathfrak{g}$ est l'ensemble des matrices carrées $X$ d'ordre $n+1$ telles que ${ }^{t} X I_{1, n}+I_{1, n} X=0$. 
- Comme sous-groupe compact maximal, on peut choisir le sous-groupe $K$ de $S O_{o}(1, n)$ formé des matrices de la forme

$$
\left(\begin{array}{ll}
1 & 0 \\
0 & k
\end{array}\right) \text {, }
$$

où $k$ est une matrice orthogonale d'ordre $n$ ( i-e telle que ${ }^{t} k k=I$ ).

- Comme sous-algèbre de Cartan $\mathfrak{a}$, on peut choisir l'ensemble des matrices de la forme

$$
\alpha_{t}:=\left(\begin{array}{ll}
0 & t \\
t & 0
\end{array}\right),
$$

avec $t \in \mathbb{R}$. On identifie $\mathfrak{a}$ avec $\mathbb{R}$.

- Comme chambre de Weyl ouverte $\mathfrak{a}^{++}$, on peut choisir le sous-ensemble de $\mathfrak{a}$ formé des éléments $t$ tel que $t>0$.

- Avec ces choix, le groupe $N$ correspond à l'ensemble des matrices $n_{u}$ de la forme

$$
n_{u}:=\left(\begin{array}{ccc}
1 & u & -\frac{\|u\|^{2}}{2} \\
0 & I_{n-1} & { }^{t} u \\
0 & 0 & 1
\end{array}\right),
$$

où $u$ est une matrice de type $1 \times(n-1)$.

- Le groupe $M$ correspond donc à l'ensemble des matrices $m_{k}$ de la forme

$$
m_{k}:=\left(\begin{array}{ccc}
1 & 0 & 0 \\
0 & k & 0 \\
0 & 0 & 1
\end{array}\right),
$$

où $k$ est une matrice orthogonale d'ordre $n-1$.

- La composante de Cartan $\mu(g) \in \mathbb{R}^{+}$d'un élément $g \in S O_{o}(1, n)$ correspond au logarithme du rayon spectral de la matrice symétrique $\left({ }^{t} g g\right)^{\frac{1}{2}}$.

(2) Explicitons certains de ces objets dans le cas où $G$ désigne le groupe spécial linéaire $S L(n, \mathbb{R})$. L'algèbre de Lie $\mathfrak{g}$ est alors l'ensemble des matrices carrées d'ordre $n$ à trace nulle.

- Comme sous-groupe compact maximal, on peut fixer le groupe orthogonal $S O(n)$.

- Comme sous-algèbre de Cartan $\mathfrak{a}$, on peut choisir l'ensemble des matrices carrées d'ordre $n$, diagonales et de trace nulle que l'on identifie avec l'hyperplan $\left\{\left(x_{1}, \ldots, x_{n}\right) \in \mathbb{R}^{n} / x_{1}+\ldots+x_{n}=0\right\}$.

- Comme chambre de Weyl ouverte $\mathfrak{a}^{++}$, on peut choisir le sous-ensemble de $\mathfrak{a}$ formé des éléments $\left(x_{1}, \ldots, x_{n}\right)$ tels que $x_{1}>\ldots>x_{n}$.

- Avec ces choix, le sous-groupe $N$ correspond au sous-groupe de $G$ formé des matrices triangulaires supérieures avec 1 sur la diagonale. 
- Avec ces choix, le sous-groupe $M$ correspond au sous-groupe de $G$ formé des matrices diagonales dont les coefficients diagonaux sont \pm 1 .

- La composante de Cartan $\mu(g) \in \mathfrak{a}^{+}$d'un élément $g \in S L(n, \mathbb{R})$ correspond au vecteur $\left(\mu_{1}(g), \ldots, \mu_{n}(g)\right) \in \mathfrak{a}$ dont les coordonnées sont les logarithmes des valeurs propres de la matrice symétrique $\left({ }^{t} g g\right)^{\frac{1}{2}}$ rangé par ordre décroissant.

Notations relatives aux espaces métriques. — Si $\left(\Xi, d_{\Xi}\right)$ est un espace métrique alors, pour tout $\xi \in \Xi$ et tout $r \in] 0,+\infty\left[\right.$, on note $\mathbb{B}_{\Xi}(\xi, r)=\left\{\zeta \in \Xi / d_{\Xi}(\xi, \zeta) \leq\right.$ $r\}$ la boule fermée centrée en $\xi$ de rayon $r$.

Notations relatives aux espaces de fonctions. - Si $\Xi$ désigne un espace topologique compact (resp localement compact), on note $C(\Xi, \mathbb{K})$ (resp. $\left.C_{c}(\Xi, \mathbb{K})\right)$, avec $\mathbb{K}=\mathbb{R}, \mathbb{R}^{+}$ou $\mathbb{C}$, l'espace des fonctions continues (resp. continues à support compact) muni de la norme uniforme $\|\cdot\|_{\infty}$.

Si $\Xi$ désigne un espace topologique localement compact, pour tout $\xi \in \Xi$, on note $\mathcal{D}_{\xi}$ la mesure de Dirac en $\xi$.

Si $\Xi$ désigne un espace mesurable, on note $\mathcal{B}_{\infty}(\Xi, \mathbb{K})$, avec $\mathbb{K}=\mathbb{R}, \mathbb{R}^{+}$ou $\mathbb{C}$, l'espace des fonctions $\phi: \Xi \rightarrow \mathbb{K}$ boréliennes et bornées muni de la norme uniforme $\|\cdot\|_{\infty}$.

Si $\Xi$ désigne un espace mesurable et $B$ un borélien de $\Xi$, on note $1_{B}$ la fonction indicatrice de $B$.

Notations relatives aux espaces euclidiens. - Si $E$ désigne un espace euclidien autre que a, on désigne par $\langle., .\rangle_{E}$ le produit scalaire associé à $E$. On note $G L(E)$ le groupe des automorphismes linéaires de $E$ et End $(E)$ l'espace des endomorphismes linéaires de $E$. Pour tout $T \in \operatorname{End}(E)$, on note $\lambda_{E}(T)$ le rayon spectral de $T$ dans $E$. On note $\wedge^{2} E$ le produit tensoriel antisymétrique de $E$ muni du produit scalaire $\langle.,.\rangle \wedge^{2} E$ défini pour $x_{1} \wedge x_{2}, y_{1} \wedge y_{2} \in \bigwedge^{2} E$ par

$$
\left\langle x_{1} \wedge x_{2}, y_{1} \wedge y_{2}\right\rangle^{2} \wedge^{2}:=\left\langle x_{1}, y_{1}\right\rangle_{E}\left\langle x_{2}, y_{2}\right\rangle_{E}-\left\langle x_{1}, y_{2}\right\rangle_{E}\left\langle x_{2}, y_{1}\right\rangle_{E}
$$

On note $\mathbb{P}(E)$ l'espace projectif associé à $E$ muni de la distance $d_{\mathbb{P}(E)}$ définie pour $\mathbb{P}(x), \mathbb{P}(y) \in \mathbb{P}(E)$ par

$$
d_{\mathbb{P}(E)}(\mathbb{P}(x), \mathbb{P}(y)):=\frac{\|x \wedge y\|_{\bigwedge^{2} E}}{\|x\|_{E}\|y\|_{E}},
$$

où $x$ et $y$ désignent respectivement un représentant de $\mathbb{P}(x), \mathbb{P}(y)$. On considère également l'action naturelle du groupe $G L(E)$ sur $\mathbb{P}(E)$ qui à $g \in G L(E)$ et $\mathbb{P}(x) \in \mathbb{P}(E)$ représenté par $x \in E$ associe $g \cdot \mathbb{P}(x)$ représenté par $g(x) \in E$.

TOME $137-2009-\mathrm{N}^{\circ} 3$ 


\section{Préliminaires}

Dans cette section, nous rassemblons des résultats connus dont nous aurons besoin par la suite.

3.1. Variété des drapeaux et cocycle de Busemann. - On rappelle que $P$ désigne le groupe $M A N$. L'espace homogène $\mathcal{F}:=G / P$ est appelé variété drapeau de $G$ et ses éléments sont appelés drapeaux de $G$.

En utilisant le fait que la décomposition d'Iwasawa est un difféomorphisme de $K \times \mathfrak{a} \times N$ dans $G$, on montre que le groupe $K$ opère transitivement sur $\mathcal{F}$ et que le stabilisateur de $P$ sous l'action de $K$ est le groupe $M$. Nous en déduisons que l'espace $\mathcal{F}$ est compact, métrisable puisque l'application $K / M \rightarrow$ $\mathcal{F}, k M \mapsto k P$ est un homéomorphisme $K$-équivariant.

Exemples. - (1) La variété des drapeaux de $S O_{o}(1, n)$ s'identifie avec le bord géométrique $\partial X$ de $X$ muni de l'action naturelle de $G$.

(2) La variété des drapeaux de $S L(n, \mathbb{R})$ s'identifie avec l'espace des drapeaux complets de $\mathbb{R}^{n}$ muni de la topologie de Hausdorff et de l'action naturelle de $G$, c'est-à-dire l'ensemble des suites $\left(E_{i}\right)_{i \in\{1, \ldots, n-1\}}$ strictement croissantes de sous-espaces vectoriels de $\mathbb{R}^{n}$ telles que $\operatorname{dim}\left(E_{i}\right)=i$ pour tout $i \in\{1, \ldots, n-1\}$.

La composante en $\mathfrak{a}$ de la décomposition d'Iwasawa d'un élément de $g \in G$ ne dépend de $g$ que par sa classe d'équivalence à droite modulo $M$. Autrement dit, si $g_{1}$ et $g_{2}$ sont deux éléments de $G$ tels que $g_{1}^{-1} g_{2} \in M$, que l'on écrit respectivement sous la forme $k_{1} \exp \left(\beta_{1}\right) n_{1}$ et $k_{2} \exp \left(\beta_{2}\right) n_{2}$ alors $\beta_{1}=\beta_{2}$. Ceci nous permet d'introduire la

Notation 3.1. - Soient $g \in G$ et $\eta \in \mathcal{F}$. On note $\beta(g, \eta)$ l'unique élément de a tel que $g k \in K \exp (\beta(g, \eta)) N$, où $k$ est un élément de $K$ tel que $\eta=k P$.

Nous rappelons la

DÉfinition-Proposition 3.2. - (Paragraphe 6 et lemme 6.2 de [12]) L'application $\beta: G \times \mathcal{F}$ qui au couple $(g, \eta)$ associe le vecteur $\beta(g, \eta)$ est appelée cocycle de Busemann et vérifie la propriété de cocyle : pour tout $(g, h) \in G \times G$ et tout $\eta \in \mathcal{F}$, on a $\beta(g h, \eta)=\beta(g, h \cdot \eta)+\beta(h, \eta)$.

\subsection{Espace des couples de drapeaux en position générale et produit de Gromov. -}

Dans ce paragraphe, nous rappelons la définition et les principales propriétés du produit de Gromov. Pour ce faire, nous introduisons les

Notations 3.3. - On fixe un élément $k_{\iota} \in K$ tel que $\operatorname{Ad}\left(k_{\iota}\right) \mathfrak{a}^{+}=-\mathfrak{a}^{+}$. De plus, on pose $\eta_{0}=P$ et $\eta_{0}^{\vee}=k_{\iota} P$. 
Exemples. - Explicitons les drapeaux $\eta_{0}$ et $\eta_{0}^{\vee}$ dans le cas où $G=S O_{o}(1, n)$ et $G=S L(n, \mathbb{R})$ avec les identifications décrites précédemment.

(1) Dans le cas où $G=S O_{o}(1, n)$, on note o le point de $\mathbb{H}^{n}$ stabilisé par $K$ et on considère la géodésique $c$ de $\mathbb{H}^{n}$ définie pour $t \in \mathbb{R}$ par $c(t)=\exp (t) \cdot o$. Avec cette notation, on a $\eta_{0}=c(+\infty)$ et $\eta_{0}^{\vee}=c(-\infty)$.

(2) Dans le cas où $G=S L(n, \mathbb{R})$, on note $\left(e_{i}\right)_{1 \leq i \leq n}$ la base usuelle de $\mathbb{R}^{n}$. Avec cette notation, on a $\eta_{0}=\left(E_{i}\right)_{1 \leq i \leq n-1}$ et $\eta_{0}^{\bar{v}}=\left(E_{n-i}^{\perp}\right)_{1 \leq i \leq n-1}$, où $E_{i}$ désigne le sous-espace de $\mathbb{R}^{n}$ engendré par l'ensemble $\left\{e_{1}, \ldots, e_{i}\right\}$ et $E_{n-i}^{\perp}$ l'orthogonal de $E_{n-i}$ dans $\mathbb{R}^{n}$ pour le produit scalaire usuel de $\mathbb{R}^{n}$.

On dit que deux drapeaux $\eta_{-}, \eta_{+} \in \mathcal{F}$ sont en position générale s'il existe $g \in G$ tel que $\eta_{-}=g \cdot \eta_{0}^{\vee}$ et $\eta_{+}=g \cdot \eta_{0}$.

Exemples. - (1) Deux drapeaux $\eta_{-}$et $\eta_{+}$de $S O_{o}(1, n)$ sont en position générale si et seulement si $\eta_{-} \neq \eta_{+}$.

(2) Deux drapeaux $\eta_{-}=\left(E_{i}^{-}\right)_{i \in\{1, \ldots, n-1\}}$ et $\eta_{+}=\left(E_{i}^{+}\right)_{i \in\{1, \ldots, n-1\}}$ de $S L(n, \mathbb{R})$ sont en position générale si et seulement si, pour tout $i \in\{1, \ldots, n-1\}$, on a $E_{i}^{-} \oplus E_{n-i}^{+}=\mathbb{R}^{n}$.

On introduit alors la

Notations 3.4. - Nous notons $\partial^{2} \mathcal{F}$ l'ensemble des couples de drapeaux en position générale, muni de la topologie induite par la topologie produit de $\mathcal{F} \times \mathcal{F}$. De plus, nous munissons $\partial^{2} \mathcal{F}$ de l'action diagonale de $G$. Si $S$ est un sousensemble de $\mathcal{F}$, nous notons $\partial^{2} S$ le sous-ensemble de $S \times S$ formé des couples de drapeaux en position générale.

Par définition de $\mathcal{F}$, le stabilisateur $\operatorname{Stab}(\eta)$ d'un drapeau $\eta=g P$ sous l'action de $G$ est le sous-groupe $g P g^{-1}$ si bien que

$$
\operatorname{Stab}\left(\eta_{0}\right) \cap \operatorname{Stab}\left(\eta_{0}^{\vee}\right)=P \cap k_{\iota} P k_{\iota}^{-1}=A M .
$$

Le stabilisateur de $\left(\eta_{0}^{\vee}, \eta_{0}\right) \in \partial^{2} \mathcal{F}$ sous l'action de $G$ est donc le groupe $A M$ et l'application $G / A M \rightarrow \partial^{2} \mathcal{F}, g A M \mapsto g \cdot\left(\eta_{0}^{\vee}, \eta_{0}\right)$ définit un homéomorphisme $G$-équivariant qui nous permet d'identifier ces deux espaces.

Nous sommes à présent en mesure d'introduire un analogue du produit de Gromov pour les espaces symétriques de type non compact. Remarquons que, si $\tau$ est un vecteur de $\mathfrak{a}$ tel que $\iota(\tau)=\tau$ alors le vecteur $\left\langle\tau, \beta\left(g, \eta_{0}^{\vee}\right)+\beta\left(g, \eta_{0}\right)\right\rangle$ ne dépend de $g$ que par sa classe d'équivalence $g A M$. Ainsi, nous pouvons introduire la

DÉFinition 3.5. - Soit $\tau \in \mathfrak{a}$ tel que $\iota(\tau)=\tau$. On appelle produit de Gromov en $\tau$ d'un élément $\left(\eta_{-}, \eta_{+}\right)=g A M \in \partial^{2} \mathcal{F}$ la quantité

$$
\left[\eta_{-}, \eta_{+}\right]_{\tau}:=\left\langle\tau, \beta\left(g, \eta_{0}^{\vee}\right)+\beta\left(g, \eta_{0}\right)\right\rangle .
$$


Par la suite, nous aurons besoin du résultat suivant qui résulte du caractère lisse de la décomposition d'Iwasawa.

Proposition 3.6. - Soit $\tau \in \mathfrak{a}$ tel que $\iota(\tau)=\tau$. L'application $\partial^{2} \mathcal{F} \rightarrow \mathbb{R}$, $\left(\eta_{-}, \eta_{+}\right) \mapsto\left[\eta_{-}, \eta_{+}\right]_{\tau}$ de classe $C^{\infty}$.

3.3. Représentations de $G$. - Soit $\rho$ une représentation rationnelle de $G$ sur un espace euclidien $E$. On dit que $\rho$ est adaptée si, pour tout $k \in K$, l'automorphisme $\rho(k)$ est orthogonal et, pour tout $\alpha \in \mathfrak{a}, \rho\left(e^{\alpha}\right)$ est symétrique. On note $d \rho$ sa différentielle. Les formes linéaires $\chi$ de $\mathfrak{a}$ pour lesquelles le sous-espace $E_{\chi}:=\{x \in E / \forall \alpha \in \mathfrak{a} \quad d \rho(\alpha) x=\chi(\alpha) x\}$ n'est pas nul sont appelées poids restreints de $\rho$. Classiquement, on munit l'ensemble $\Sigma(\rho)$ des poids restreints de $\rho$ de la relation d'ordre $\leq$ définie par

$$
\left(\chi_{1} \leq \chi_{2}\right) \Longleftrightarrow\left(\forall \alpha \in \mathfrak{a}^{+} \quad \chi_{1}(\alpha) \leq \chi_{2}(\alpha)\right) .
$$

Lorsque la représentation $\rho$ est irréductible, l'ensemble $\Sigma(\rho)$ possède un unique élément maximal (pour la relation d'ordre $\leq$ ) appelé plus haut poids restreint de $\rho$. Dans ce cas, la représentation $\rho$ est dite proximale si $\operatorname{dim}\left(E_{\chi}\right)=1$, où $\chi$ désigne le plus haut poids restreint de $\rho$.

Exemples. - Donnons quelques exemples de plus hauts poids restreints.

(1) La représentation usuelle de $S O_{o}(1, n)$ sur $\mathbb{R}^{n+1}$ est irréductible, rationnelle et proximale. Son plus haut poids restreint $\chi$ est la forme linéaire sur $\mathfrak{a}$ définie pour $\alpha \in \mathfrak{a}$ par $\chi(\alpha)=\alpha$ et l'on a $E_{\chi}^{+}=\left\{x\left(e_{1}+e_{n+1}\right) / x \in \mathbb{R}\right\}$, où $\left(e_{i}\right)_{1 \leq i \leq n+1}$ désigne la base usuelle de $\mathbb{R}^{n+1}$.

(2) Pour tout $i \in\{1, \ldots, n-1\}$, on note $\bigwedge^{i}$ la représentation usuelle de $S L(n, \mathbb{R})$ sur $\bigwedge^{i} \mathbb{R}^{n}$. C'est une représentation rationnelle et irréductible de $G$ dont le plus haut poids restreint $\chi_{i}$ est la forme linéaire sur a définie pour $\alpha=\left(\alpha_{1}, \ldots, \alpha_{n}\right) \in \mathfrak{a}$ par $\chi_{i}(\alpha)=\alpha_{1}+\ldots+\alpha_{i}$. De plus, $\bigwedge^{i}$ est proximale car le sous-espace $E_{\chi_{i}}$ est engendré par le vecteur $e_{1} \wedge \ldots \wedge e_{i}$, où $\left(e_{j}\right)_{1 \leq j \leq n}$ désigne la base canonique de $\mathbb{R}^{n}$.

La définition de groupe de Ping-Pong sur $\mathcal{F}$ que nous avons proposée dans [15] s'appuie sur la théorie des représentations. On utilise essentiellement le fait qu'il existe une famille finie de représentations dont les plus hauts poids restreints permettent de calculer les éléments de a. Aussi, nous rappelons la

Proposition 3.7. - (Lemmes 2.1 et 2.2 de [6]) Il existe une famille de représentations $\left(\rho_{\delta}: G \rightarrow G L\left(E_{\delta}\right) / \delta \in \Delta\right)$ irréductibles, rationnelles, proximales et adaptées telles que l'application

$$
\mathfrak{a} \rightarrow \mathbb{R}^{\Delta} \quad \alpha \mapsto\left(\chi_{\delta}(\alpha)\right)_{\delta \in \Delta}
$$


où $\chi_{\delta}$ désigne le plus haut poids restreint de $\rho_{\delta}$, soit un isomorphisme d'espaces vectoriels.

3.3.1. Exemples. - (1) Dans le cas où $G$ désigne le groupe orthochrone $S O_{o}(1, n)$, la représentation usuelle de $S O_{o}(1, n)$ sur $\mathbb{R}^{n+1}$ convient puisque l'application $\mathfrak{a} \rightarrow \mathbb{R}, \alpha \mapsto \chi(\alpha)$ est un isomorphisme.

(2) Dans le cas où $G$ désigne le groupe spécial linéaire $S L(n, \mathbb{R})$, la famille de représentations $\left(\bigwedge^{k} / k \in\{1, \ldots, n-1\}\right)$ convient puisque l'application $\mathfrak{a} \rightarrow$ $\mathbb{R}^{n-1},\left(\alpha_{1}, \ldots \alpha_{n}\right) \mapsto\left(\alpha_{1}, \ldots, \alpha_{1}+\ldots+\alpha_{n-1}\right)$ définit un isomorphisme.

\section{Sous-groupes discrets}

Nous rappelons ici une partie des résultats de [5], [9] et [11]. Pour ce faire, Fixons un sous-groupe discret et Zariski dense $\Gamma$ de $G$.

Cône limite. - Suivant la terminologie employée dans [5], le cône limite de $\Gamma$ est le plus petit cône fermé de $\mathfrak{a}^{+}$contenant $\lambda(\Gamma)=\left\{\lambda(\gamma) \in \mathfrak{a}^{+} / \gamma \in \Gamma\right\}$. Par la suite, nous aurons besoin du

ThÉORÈme 4.1. - (Théorème 1.2 de [5]) Le cône limite $l_{\Gamma}$ de $\Gamma$ est convexe et d'intérieur non vide.

Ensemble limite. - Notons $\mathcal{M}(\mathcal{F})$ l'espace des mesures boréliennes sur $\mathcal{F}$ muni de la topologie de la convergence faible-* et sur lequel le groupe $G$ opère naturellement. De plus, considérons l'unique mesure de probabilité $\nu$ sur $\mathcal{F}$ invariante pour l'action de $K$.

L'ensemble limite $\Lambda_{\Gamma}$ dans $\mathcal{F}$ de $\Gamma$ est l'ensemble des points $\eta \in \mathcal{F}$ pour lesquels il existe une suite d'éléments $\left(\gamma_{n}\right)_{n \in \mathbb{N}}$ de $\Gamma$ telle que $\left(\gamma_{n} \cdot \nu\right)_{n \in \mathbb{N}}$ converge dans $\mathcal{M}(\mathcal{F})$ vers la mesure de Dirac en $\eta$.

ThÉorème 4.2. - ([9],Lemme 3.6 de [5]) L'ensemble limite $\Lambda_{\Gamma}$ dans $\mathcal{F}$ de $\Gamma$ est la seule partie fermée de $\mathcal{F}$ qui soit minimale pour l'action de $\Gamma$.

Indicateur de croissance et vecteur de croissance. - Si $\lambda$ est une mesure de Radon sur un espace normé $E$, la borne inférieure $\chi_{\lambda}$ de l'ensemble $\{s \in$ $\left.\mathbb{R}^{+} / \int_{E} e^{-s\|x\|_{E}} d \lambda(x)<+\infty\right\}$ est appelée exposant critique de $\lambda$. D'après le lemme 3.1.1 de [11], si $\chi_{\lambda}>0$ alors on a

$$
\chi_{\lambda}=\limsup _{R \rightarrow+\infty} \frac{\ln (\lambda([0, R]))}{R} .
$$

Pour tout cône ouvert $\mathcal{C}$ de $\mathfrak{a}^{+}$, on note $\delta_{\Gamma, \mathcal{C}}$ l'exposant critique de la mesure

$$
\sum_{\{\gamma \in \Gamma / \mu(\gamma) \in \mathcal{C}\}} \mathcal{D}_{d_{X}(o, \gamma \cdot o)} .
$$

TOME $137-2009-\mathrm{N}^{\circ} 3$ 
Suivant la terminologie employée dans [11], l'indicateur de croissance de $\Gamma$ est l'application $\Psi_{\Gamma}: \mathfrak{a}^{+} \rightarrow \mathbb{R} \cup\{-\infty\}$ telle que $\Psi_{\Gamma}(0)=0$ et qui à $\alpha \in \mathfrak{a}^{+} \backslash\{0\}$ associe

$$
\|\alpha\| \inf \delta_{\Gamma, \mathcal{C}}
$$

la borne inférieure étant prise sur l'ensemble des cônes ouverts de $\mathfrak{a}^{+}$contenant $\alpha$. Par la suite, nous aurons besoin du résultat suivant démontré dans [11].

THÉORÈme 4.3. - (Théorème 4.2.2 de [11]) L'indicateur de croissance $\Psi_{\Gamma}$ d'un sous-groupe discret et Zariski dense est homogène, concave et semi-continu supérieurement. De plus, l'ensemble

$$
\left\{\alpha \in \mathfrak{a}^{+} / \Psi_{\Gamma}(\alpha)>-\infty\right\}
$$

est exactement le cône limite $l_{\Gamma}$ de $\Gamma$.

D'après ce théorème l'indicateur de croissance de $\Gamma$ est concave et homogène sur $\mathfrak{a}^{+}$et donc, on a $\delta_{\Gamma}=\sup _{v \in l_{\Gamma},\|v\|=1} \Psi_{\Gamma}(v)$ et il existe un unique vecteur unitaire $u \in l_{\Gamma}$ tel que $\Psi_{\Gamma}(u)=\delta_{\Gamma}$.

Proposition 4.4. - (Corollaire 4.2.4 de [11]) Si u désigne l'unique vecteur unitaire de $l_{\Gamma}$ tel que $\Psi_{\Gamma}(u)=\delta_{\Gamma}$ alors la série $\sum_{\gamma \in \Gamma} e^{-s\langle u, \mu(\gamma)\rangle}$ converge si $s>\Psi_{\Gamma}(u)$ et diverge si $s<\Psi_{\Gamma}(u)$

Le vecteur $\tau(\Gamma):=\Psi(u) u$ sera appelé vecteur de croissance de $\Gamma$.

\section{Transformations contractantes sur $\mathcal{F}$}

La construction des groupes de Schottky employée par Y. Benoist dans [5] s'appuie sur une classe particulière d'éléments de $G$, les transformations loxodromiques. Ces transformations peuvent être vues comme l'analogue en rang supérieur des transformations hyperboliques d'un groupe de rang 1. Aussi, afin d'étendre la construction étudiée par F. Dal'bo et M. Peigné dans [7], nous considérerons une classe de transformations, les transformations contractantes sur $\mathcal{F}$, qui pourra être vue comme l'analogue en rang supérieur des transformations hyperboliques ou paraboliques. Pour cela, nous avons besoin d'introduire les :

Notations 5.1. - On fixe à présent une famille de représentations $\left(\rho_{\delta}: G \rightarrow\right.$ $\left.G L\left(E_{\delta}\right) / \delta \in \Delta\right)$ irréductibles, proximales et adaptées comme dans la proposition 3.7. De plus, pour tout $\delta \in \Delta$, on note

- $\chi_{\delta}$ le plus haut poids restreint de $G$.

- $E_{\delta}^{+}$la droite de $E_{\delta}$ définie par $E_{\delta}^{+}=\left\{x \in E_{\delta} / \forall \alpha \in \mathfrak{a} \quad d \rho_{\delta}(\alpha) x=\right.$ $\left.\chi_{\delta}(\alpha) x\right\}$.

- $\mathbb{P}\left(x_{\delta}^{+}\right)$l'élément de $\mathbb{P}\left(E_{\delta}\right)$ représenté par un vecteur de $E_{\delta}^{+} \backslash\{0\}$. 
- $\pi_{\delta}$ l'application de $\mathcal{F}$ sur $\mathbb{P}\left(E_{\delta}\right)$ définie pour gP par $\pi_{\delta}(g P)=\rho_{\delta}(g)$. $\mathbb{P}\left(x_{\delta}^{+}\right)$.

De plus, on munit la variété des drapeaux $\mathcal{F}(=G / P)$ de la distance $d_{\mathcal{F}}$ définie pour $\eta_{1}, \eta_{2} \in \mathcal{F}$ par $d_{\mathcal{F}}\left(\eta_{1}, \eta_{2}\right)=\sum_{\delta \in \Delta} d_{\mathbb{P}\left(E_{\delta}\right)}\left(\pi_{\delta}\left(\eta_{1}\right), \pi_{\delta}\left(\eta_{2}\right)\right)$.

Commençons par rappeler la

DÉfinition 5.2. - (Définition 3.1 de [6]) Un élément $g \in G$ est dit loxodromique si $\lambda(g) \in \mathfrak{a}^{++}$.

Remarque. - Pour toute transformation loxodromique $g \in G$, il existe un unique $\left(\eta_{g}^{-}, \eta_{g}^{+}\right) \in \partial^{2} \mathcal{F}$ tel que, pour tout $\eta \in \mathcal{F}$ en position générale avec $\eta_{g}^{-}$, la suite $\left(g^{n} \cdot \eta\right)_{n \in \mathbb{N}}$ converge vers $\eta_{g}^{+}$. En particulier, si le rang de $G$ vaut 1 , une transformation $g \in G$ est loxodromique si et seulement si l'isométrie de $X$ qui lui est associée est hyperbolique.

Le fait qu'un élément $g \in G$ soit loxodromique s'exprime également par le biais de la famille de représentations $\left(\rho_{\delta}: G \rightarrow G L\left(E_{\delta}\right) / \delta \in \Delta\right)$. En effet, rappelons qu'un automorphisme $g$ d'un espace vectoriel $E$ est dit proximal s'il admet une unique valeur propre de module $\lambda_{E}(g)$ et si cette valeur propre est simple. En fait, il est équivalent de dire qu'un élément $g \in G$ est loxodromique si, pour tout $\delta \in \Delta$, l'automorphisme $\rho_{\delta}(g)$ est proximal. Ceci nous amène a introduire la

\section{DÉFINITIONS 5.3}

- Soit $E$ un espace euclidien et soit $g \in G L(E)$. On dit que $g$ est contractant sur $\mathbb{P}(E)$ s'il existe $\mathbb{P}\left(x_{g}^{+}\right) \in \mathbb{P}(E)$, dit droite attractive de $g$, et un hyperplan projectif $\mathbb{P}\left(X_{g}\right) \subset \mathbb{P}(E)$, dit hyperplan répulsif, tels que, pour tout $\mathbb{P}(x) \notin \mathbb{P}\left(X_{g}\right)$, la suite $\left(g^{n} \cdot \mathbb{P}(x)\right)_{n \in \mathbb{N}}$ converge vers $\mathbb{P}\left(x_{g}^{+}\right)$.

- Un élément $g \in G$ est dit contractant sur $\mathcal{F}$ si, pour tout $\delta \in \Delta$, l'automorphisme $\rho_{\delta}(g)$ est contractant sur $\mathbb{P}\left(E_{\delta}\right)$.

REMARques. - (1) Lorsque $r g(G)=1$, une transformation est contractante sur $\mathcal{F}$ si et seulement si l'isométrie de $X$ qui lui est associée est parabolique ou hyperbolique.

(2) Il existe des transformations contractantes sur $\mathcal{F}$ qui ne sont pas loxodromiques. Par exemple si $G=S L(n, \mathbb{R})$, toute matrice unipotente $g=I+U$, où $I$ désigne la matrice identité et $U$ un endomorphisme nilpotent tel que $U^{n-1} \neq 0$, est un élément de $G$ contractant sur $\mathcal{F}$ qui n'est pas loxodromique.

Nous utiliserons les

Notations 5.4. - Soit $\varpi$ un nombre réel appartenant à ] 0,1 [ et soit $g \in G$ une transformation contractante sur $\mathcal{F}$. 
- Pour tout $\delta \in \Delta$, on note $\mathbb{P}\left(x_{\delta, g}^{+}\right) \in \mathbb{P}\left(E_{\delta}\right)$ la droite attractive de $\rho_{\delta}(g)$ et $\mathbb{P}\left(X_{\delta, g}\right) \subset \mathbb{P}\left(E_{\delta}\right)$ l'hyperplan répulsif de $\rho_{\delta}(g)$.

- On note $\mathbf{b}_{g}^{\varpi}$ la partie de $\mathcal{F}$, appelée bassin d'arrivée, définie par

$$
\mathbf{b}_{g}^{\varpi}:=\left\{\eta \in \mathcal{F} / \forall \delta \in \Delta \quad d_{\mathbb{P}\left(E_{\delta}\right)}\left(\mathbb{P}\left(x_{\delta, g}^{+}\right), \pi_{\delta}(\eta)\right) \leq \varpi\right\}
$$

- On note $\mathbf{B}_{g}^{\varpi}$ la partie de $\mathcal{F}$, appelée bassin de départ, définie par

$$
\mathbf{B}_{g}^{\varpi}:=\left\{\eta \in \mathcal{F} / \forall \delta \in \Delta \quad d_{\mathbb{P}\left(E_{\delta}\right)}\left(\mathbb{P}\left(X_{\delta, g}\right), \pi_{\delta}(\eta)\right) \geq \varpi\right\} .
$$

Le résultat qui suit précise la «dynamique » d'une transformation contractante sur $\mathcal{F}$.

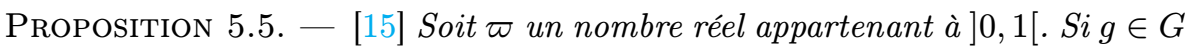
est contractant sur $\mathcal{F}$ alors $\mathbf{B}_{g}^{\varpi}$ est d'intérieur non vide dans $\mathcal{F}$ et il existe un unique drapeau $\eta_{g}^{+} \in \mathcal{F}$, appelé drapeau attractif, tel que, pour tout $\eta \in \mathbf{B}_{g}^{\varpi}$, la suite $\left(g^{n} \cdot \eta\right)_{n \in \mathbb{N}}$ converge vers $\eta_{g}^{+}$. De plus, pour tout $\delta \in \Delta$, on a $\pi_{\delta}\left(\eta_{g}^{+}\right)=$ $\mathbb{P}\left(x_{\delta, g}^{+}\right)$.

\section{Nous utiliserons la}

Notation 5.6. - Sig est un élément de $G$ qui est $\varpi$-contractant sur $\mathcal{F}$, nous notons $\eta_{g}^{+}$son drapeau attractif.

Pour notre étude, nous aurons besoin d'une propriété plus forte que la contraction. En effet, il nous faudra considérer des éléments de $G$ contractant sur $\mathcal{F}$ dont nous pouvons «quantifier» la dynamique. Aussi, nous introduisons les

Notations 5.7. - Soient $E$ un espace euclidien, $\epsilon \in] 0,1[$ et $g$ un automorphisme linéaire de $E$ contractant sur $\mathbb{P}(E)$ de droite attractive $\mathbb{P}\left(x_{g}\right)$ et d'hyperplan répulsif $\mathbb{P}\left(X_{g}\right)$. On note

- $\mathbf{b}_{g}^{\epsilon}$ la partie de $\mathbb{P}(E)$ définie par

$$
\mathbf{b}_{g}^{\epsilon}:=\left\{\mathbb{P}(x) \in \mathbb{P}(E) / d_{\mathbb{P}(E)}\left(\mathbb{P}\left(x_{g}\right), \mathbb{P}(x)\right) \leq \epsilon\right\}
$$

- $\mathbf{B}_{g}^{\epsilon}$ la partie de $\mathbb{P}(E)$ définie par

$$
\mathbf{B}_{g}^{\epsilon}:=\left\{\mathbb{P}(x) \in \mathbb{P}(E) / d_{\mathbb{P}(E)}\left(\mathbb{P}\left(X_{g}\right), \mathbb{P}(x)\right) \geq \epsilon\right\} .
$$

DÉFInItion 5.8. - Soit E un espace euclidien. On dit qu'un automorphisme linéaire $g$ est $\epsilon$-contractant sur $\mathbb{P}(E)$ si, pour tout $n \geq 1$,

- on a $g^{n} \cdot \mathbf{B}_{g}^{\epsilon} \subset \mathbf{b}_{g}^{\epsilon}$

- la restriction de $g^{n} \grave{a} \mathbf{B}_{g}^{\epsilon}$ est $\epsilon$-lipschitzienne

Nous rappelons la 
DÉfinition 5.9. - [15] Soit $\varpi \in] 0,1[$. Un élément $g \in G$ sera dit $\varpi-$ contractant sur $\mathcal{F}$ si, pour tout $\delta \in \Delta$, l'automorphisme $\rho_{\delta}(g)$ est $\varpi$-contractant sur $\mathbb{P}\left(E_{\delta}\right)$.

\section{Groupes de Ping-Pong}

Nous rappelons ici une partie des résultats de [15] concernant les groupes de Ping-Pong sur $\mathcal{F}$.

\subsection{Définition des groupes de Ping-Pong. - Nous rappelons la}

DÉFINITION 6.1. - [15] Soit $\varpi$ un nombre réel appartenant à ]0,1[. Une partie $\mathcal{A}:=\left\{\gamma_{1}^{ \pm 1}, \ldots, \gamma_{N}^{ \pm 1}\right\}$, avec $N \geq 2$, d'éléments $\varpi$-contractants sur $\mathcal{F}$ de $G$ est dite en position $\varpi$-Ping-Pong si

1. pour tous $a_{1}, a_{2} \in \mathcal{A}$ avec $a_{1} \neq a_{2}^{ \pm 1}$, l'ensemble $\mathbf{b}_{a_{1}}$ est inclu dans l'intérieur de $\mathbf{B}_{a_{2}}$.

2. pour tous $a_{1}, a_{2} \in \mathcal{A}$ avec $a_{1} \neq a_{2}^{ \pm 1}$, l'ensemble $\mathbf{b}_{a_{1}} \times \mathbf{b}_{a_{2}}$ est contenu dans $\partial^{2} \mathcal{F}$.

3. L'ensemble $\mathbf{B}_{\Gamma}:=\cap_{a \in \mathcal{A}} \mathbf{B}_{a}$ est non vide.

Le sous-groupe de $G$ engendré par une partie $\mathcal{A}$ en position Ping-Pong est appelé groupe $\varpi$-Ping-Pong sur $\mathcal{F}$ engendré par $\mathcal{A}$.

REMARQUes. - (1) Lorsque tous les éléments d'une partie $\mathcal{A}$, symétrique en position $\varpi-P i n g-P o n g$, sont loxodromiques, suivant la terminologie utilisée dans [13], on dit que le groupe engendré par $\mathcal{A}$ est de type Schottky.

(2) Lorsque $X$ désigne l'espace hyperbolique de dimension $n$, l'espace $\partial^{2} \mathcal{F}$ s'identifie avec $\partial^{2} X:=\partial X \times \partial X \backslash$ diag, où $\partial X$ désigne le bord géométrique de $X$. Dans ce cas, la condition (2) signifie que les bassins d'attractions $\mathbf{b}_{a}$ et $\mathbf{b}_{a^{\prime}}$ de deux transformations a et $a^{\prime}$ de $\mathcal{A}$ telles que $a^{\prime} \neq a^{ \pm 1}$ sont deux-à-deux disjoints. D'après la remarque qui suit la définition 5.3, on retrouve les groupes de Ping-Pong introduit dans [7].

L'existence de groupe de Ping-Pong est connue puisque les groupes de Schottky sont engendrés par une partie en position Ping-Pong. Toutefois, il existe des groupes de Ping-Pong qui ne sont pas de type Schottky, il suffit pour cela d'appliquer le résultat qui suit à une famille finie et symétrique de $G$ contenant une transformation contractante et non loxodromique.

Proposition 6.2. - [15] Soit $\mathcal{A}$ une partie symétrique de $G$ formées de transformations contractantes sur $\mathcal{F}$. On suppose que, pour tous $a_{1}, a_{2} \in \mathcal{A}$ tels que $a_{1} \neq a_{2}^{ \pm 1}$, on $a\left(\eta_{a_{1}}^{+}, \eta_{a_{2}}^{+}\right) \in \partial^{2} \mathcal{F}$. Pour tout $\varpi>0$ suffisamment petit, il existe $n \in \mathbb{N}$ tel que la partie $\left\{a^{n} \in G / a \in \mathcal{A}\right\}$ soit en position $\varpi$-Ping-Pong sur $\mathcal{F}$. 
Dans tout le reste de ce travail, $\Gamma$ désignera un sous-groupe discret et Zariski dense engendré par une partie $\mathcal{A}:=\left\{\gamma_{1}^{ \pm 1}, \ldots, \gamma_{N}^{ \pm 1}\right\}$ de $G$ en position $\varpi$-PingPong avec $N \geq 3$. De plus, on notera $\tau(\Gamma)$ son vecteur de croissance.

Le résultat qui suit précise les propriétés algébriques de $\Gamma$ et repose sur le lemme du tennis de table de Klein.

Corollaire 6.3. - [15] Le groupe $\Gamma$ est libre et discret.

Ce résultat nous amène à introduire la

DÉfinition 6.4. - Une suite $\left(a_{i}, n_{i}\right)_{l_{0} \leq i \leq l}$ d'éléments de $\mathcal{A} \times \mathbb{N}^{*}$ (avec $l_{0} \in$ $\{0,1\}$ et éventuellement $l=+\infty)$ sera dite $\mathcal{A}^{*}$-admissible si, pour tout $i \in$ $\left\{l_{0}, \ldots, l-1\right\}$, on $a a_{i} \in \mathcal{A}$ et $a_{i} \neq a_{i+1}^{ \pm 1}$.

Nous notons $\Sigma_{+}$l'ensemble des suites $\mathcal{A}^{*}$-admissibles.

En effet, d'après le corollaire 6.3 , tout élément $\gamma \in \Gamma$ se décompose de façon unique sous la forme $a_{1}^{n_{1}} \ldots a_{k}^{n_{k}}$ où $\left(a_{i}, n_{i}\right)_{i \in\{1, \ldots, k\}}$ est une suite $\mathcal{A}^{*}$-admissible finie. Ainsi, nous introduisons également les

Notations 6.5. - Soit $\gamma$ une transformation de $\Gamma$ qui se décompose sous la forme $\gamma=a_{1}^{n_{1}} \ldots a_{k}^{n_{k}}$ ò̀ $\left(a_{i}, n_{i}\right)_{1 \leq i \leq k}$ est une suite $\mathcal{A}^{*}$-admissible.

- L'entier $k$ est appelé longueur de $\gamma$ et on le note $l(\gamma)$.

- La suite $\left(a_{i}, n_{i}\right)_{1<i<k}$ s'appelle $\mathcal{A}^{*}$-décomposition de $\gamma$.

- L'ensemble $\mathbf{b}_{a_{1}}^{\varpi}$ est appelé bassin d'arrivée de $\gamma$ et on le note $\mathbf{b}_{\gamma}$.

- L'ensemble $\mathbf{B}_{a_{k}}^{\varpi}$ est appelé bassin de départ de $\gamma$ et on le note $\mathbf{B}_{\gamma}$.

Pour tout $l \in \mathbb{N}$, nous notons $\Gamma^{(l)}$ le sous-ensemble de $\Gamma$ formé des éléments de longueur $l$.

6.2. Codage de l'ensemble limite. - Il a été montré dans [13] que l'ensemble limite d'un groupe de Schottky (voir [5] et [13]) s'identifie via un homéomorphisme bihöldérien avec un espace symbolique sur un alphabet fini. Dans ce paragraphe, nous rappelons comment nous avons étendu dans [15] cette identification au cas des groupes de Ping-Pong sur $\mathcal{F}$. On introduit la

Notations 6.6. - Nous notons $\Lambda_{\Gamma}$ l'ensemble limite de $\Gamma$ et nous posons $\Lambda_{\Gamma}^{0}:=\Lambda_{\Gamma} \backslash\left\{\gamma \cdot \eta_{a}^{+} / \gamma \in \Gamma, a \in \mathcal{A}\right\}$.

Nous avons la

Proposition 6.7. - [15] Avec les notations introduites ci-dessus.

1. Pour toute suite $\mathbf{a}=\left(a_{i}, n_{i}\right)_{i \in \mathbb{N}}$ de $\Sigma_{+}$, la suite $\left(\left(a_{0}^{n_{0}} \ldots a_{i}^{n_{i}}\right) \cdot \xi\right)_{i \in \mathbb{N}}$ converge vers un point $\pi_{+}(\mathbf{a})$ de $\Lambda_{\Gamma}$ qui ne dépend pas du choix de $\xi \in \mathbf{B}_{\Gamma}$.

2. L'application $\pi_{+}$de $\Sigma_{+}$dans $\Lambda_{\Gamma}$ est injective.

3. On $a \pi_{+}\left(\Sigma_{+}\right)=\Lambda_{\Gamma}^{0}$. 
Dorénavant, nous identifierons les espaces $\Lambda_{\Gamma}^{0}$ et $\Sigma_{+}$.

\section{Flot des chambres de Weyl de $\Gamma$}

Dans cette section, nous montrons que le flot des chambres de Weyl de $\Gamma$ muni de la mesure de Patterson-Sullivan est conjugué à un flot spécial défini au dessus d'un espace symbolique d'alphabet infini.

7.0.0.1. Définition du flot des chambres de Weyl. — Introduisons les

Notations 7.1. - L'espace des chambres de Weyl $\Gamma \backslash G / M$ (resp. G/M) de $\Gamma \backslash G / K($ resp. $G / K)$ est noté $\mathcal{W}_{\Gamma}($ resp. $\mathcal{W})$.

On rappelle que $M$ désigne le centralisateur de $A$ dans $K$. Ainsi, pour tout $\alpha \in \mathfrak{a}$, on a $e^{\alpha} M=M e^{\alpha}$. Cette remarque permet ainsi de définir le flot des chambres de Weyl.

DÉfinition+Notation 7.2. - Le flot des chambres de Weyl de $\mathcal{W}_{\Gamma}$ (resp. $\mathcal{W})$, que nous noterons $\left(w_{\Gamma}^{\alpha} / \alpha \in \mathfrak{a}\right)$ (resp. $\left(w_{\alpha} / \alpha \in \mathfrak{a}\right)$ ), est l'action par homéomorphisme de a sur $\mathcal{W}_{\Gamma}($ resp. $\mathcal{W})$ défini pour $\alpha \in \mathfrak{a}$ et $x=\Gamma g M$ (resp $x=g M)$ par

$$
w_{\Gamma}^{\alpha}(x)=\Gamma g e^{\alpha} M \quad\left(\text { resp. } w_{\alpha}(x)=g e^{\alpha} M\right) .
$$

Décrivons à présent l'identification de $\mathcal{W}$ avec l'espace produit $\partial^{2} \mathcal{F} \times \mathfrak{a}$ que nous utiliserons dans ce travail. On fait opérer le groupe $G$ sur $\partial^{2} \mathcal{F} \times \mathfrak{a}$ en associant à $g \in G$ et $\left(\eta_{-}, \eta_{+}, x\right) \in \partial^{2} \mathcal{F} \times \mathfrak{a}$ le point

$$
g \cdot\left(\eta_{-}, \eta_{+}, x\right)=\left(g \cdot \eta_{-}, g \cdot \eta_{+}, x-\beta\left(g, \eta_{+}\right)\right) .
$$

Cette action est transitive et le stabilisateur de $\left(\eta_{0}^{\vee}, \eta_{0}, 0\right)$ est $M$. Nous en déduisons que l'application

$$
\mathcal{W} \rightarrow \partial^{2} \mathcal{F} \times \mathfrak{a} \quad g M \mapsto\left(g \cdot \eta_{0}^{\vee}, g \cdot \eta_{0},-\beta\left(g, \eta_{0}\right)\right)
$$

est un homéomorphisme $G$-équivariant qui nous permet d'identifier ces deux espaces. Dans ce système de coordonnées, l'action du flot des chambres de Weyl est donnée par

$$
w_{\alpha}\left(\left(\eta_{-}, \eta_{+}, x\right)\right)=\left(\eta_{-}, \eta_{+}, x-\alpha\right)
$$

puisque, pour tout $\alpha \in \mathfrak{a}$, on a $\beta\left(\exp (\alpha), \eta_{0}\right)=\alpha$. 
Mesures de Patterson-Sullivan des groupes de Ping-Pong. - Dans ce paragraphe, nous utilisons les résultats de [15] concernant les mesures de Patterson sur $\mathcal{F}$ pour les groupes de Ping-Pong. Rappelons la

DÉFInItion 7.3. - [12] Soit $H$ un sous-groupe discret et Zariski dense de $G$ et soit $L$ une forme linéaire sur $\mathfrak{a}$. Une mesure borélienne $\nu$ sur $\mathcal{F}$ est une $(H, L)$-mesure de Patterson sur $\mathcal{F}$ si, pour tout $h \in H$ et tout $\phi \in C(\mathcal{F}, \mathbb{R})$, on $a$

$$
\int_{\mathcal{F}} \phi(h \cdot \eta) d \nu(\eta)=\int_{\mathcal{F}} \phi(\eta) e^{-L\left(\beta\left(h^{-1}, \eta\right)\right)} d \nu(\eta) .
$$

Par la suite, nous ne considérerons que les $(\Gamma, L)$-mesures de Patterson, où $L$ est la forme linéaire sur a définie pour $\alpha$ par $L(\alpha)=\langle\tau(\Gamma), \alpha\rangle$. Aussi, afin d'alléger le texte, nous commettrons l'abus de langage qui consiste à dire qu'une mesure borélienne $\nu$ sur $\mathcal{F}$ est de Patterson si, pour tout $\gamma \in \Gamma$ et tout $\phi \in C\left(\Lambda_{\Gamma}, \mathbb{R}\right)$, on a

$$
\int_{\Lambda_{\Gamma}} \phi(\gamma \cdot \eta) d \nu(\eta)=\int_{\Lambda_{\Gamma}} \phi(\eta) e^{-\left\langle\tau(\Gamma), \beta\left(\gamma^{-1}, \eta\right)\right\rangle} d \nu(\eta)
$$

Nous avons le

THÉORÈme 7.4. - [15] ’̀ constante multiplicative près, il existe une unique mesure de Patterson sur $\mathcal{F}$ pour $\Gamma$ dont le support topologique est $\Lambda_{\Gamma}$. De plus, si $\nu$ désigne une mesure de Patterson sur $\mathcal{F}$ dont le support topologique est $\Lambda_{\Gamma}$ alors $\Lambda_{\Gamma}^{0}$ est borélien de $\nu$-mesure pleine.

Ceci nous amène à introduire la

Notation 7.5. - Nous notons $\nu$ l'unique mesure de probabilité sur $\mathcal{F}$ qui est de Patterson pour $\Gamma$ et dont le support topologique est $\Lambda_{\Gamma}$.

Rappelons à présent la construction de la mesure de Patterson-Sullivan associée à $\nu$. Via l'identification entre $\mathcal{W}$ et $\partial^{2} \mathcal{F} \times \mathfrak{a}$, la mesure de Radon $m$ sur $\mathcal{W}$ définie par

$$
d m\left(\eta_{-}, \eta_{+}, x\right)=\frac{\left.d \nu \otimes \nu\right|_{\partial^{2} \mathcal{F}}}{e^{-\left[\eta_{-}, \eta_{+}\right]_{\tau(\Gamma)}}} \otimes d x
$$

où l'on rappelle que $\left[\eta_{-}, \eta_{+}\right]_{\tau(\Gamma)}$ désigne le produit de Gromov en $\tau(\Gamma)$ de $\left(\eta_{-}, \eta_{+}\right)$, est appelée mesure de Patterson-Sullivan sur $\mathcal{W}$ associée à $\nu$. Elle est non nulle et invariante par le flot des chambres de Weyl $\left(w_{\alpha} / \alpha \in \mathfrak{a}\right)$ et l'action à gauche de $\Gamma \operatorname{sur} \mathcal{W}(=G / M)$. Le groupe étant libre, il est sans torsion et, puisque $\Gamma$ est discret, l'action de $\Gamma$ sur $\mathcal{W}$ est propre. La projection naturelle de $\mathcal{W}(=G / M)$ sur $\mathcal{W}_{\Gamma}(=\Gamma \backslash G / M)$ est un revêtement et la mesure $m$ induit par passage au quotient une mesure $m_{\Gamma}$, appelée mesure de Patterson-Sullivan sur $\mathcal{W}_{\Gamma}$ associée $\grave{a} \nu$, invariante par $\left(w_{\Gamma}^{\alpha} / \alpha \in \mathfrak{a}\right)$. Nous utiliserons les 
Notations 7.6. - Nous notons $m_{\Gamma}$ (resp. m) la mesure de PattersonSullivan sur $\mathcal{W}_{\Gamma}$ (resp. sur $\left.\mathcal{W}\right)$ associée à $\nu$.

7.0.0.2. Le codage du flot des chambres de Weyl. - Dans ce paragraphe, nous montrons que le système dynamique mesuré $\left(\mathcal{W}_{\Gamma},\left(w_{\Gamma}^{\alpha} / \alpha \in \mathfrak{a}\right), m_{\Gamma}\right)$ est mesurablement conjugué à un espace symbolique d'alphabet infini.

Comme le borélien $\Lambda_{\Gamma}^{0}$ est de mesure $\nu$-pleine, nous pouvons restreindre $m_{\Gamma}$ à l'ensemble $\mathcal{W}_{\Gamma}^{0}$ défini ci-dessous.

Notation 7.7. - Nous notons $\mathcal{W}_{\Gamma}^{0}$ l'ensemble des points $\Gamma g M \in \mathcal{W}_{\Gamma}$ pour lesquels il existe un représentant $g=\left(\eta_{-}, \eta_{+}, x\right)$ vérifiant $\eta_{ \pm} \in \Lambda_{\Gamma}^{0}$.

Remarque. - Notons que $\mathcal{W}_{\Gamma}^{0}$ est un borélien $\left(w_{\Gamma}^{\alpha} / \alpha \in \mathfrak{a}\right)$-invariant.

Introduisons à présent les quelques objets dont nous aurons besoin

\section{Notations 7.8}

- Nous notons $\Sigma$ l'ensemble des suites $\left(a_{k}, n_{k}\right)_{k \in \mathbb{Z}}$, dites $\mathcal{A}^{*}$-admissibles, d'éléments de $\mathcal{A} \times \mathbb{N}^{*}$ telles que, pour tout $k \in \mathbb{Z}$, on ait $a_{k} \neq a_{k+1}^{ \pm 1}$. Nous notons $\theta$ le décalage sur $\Sigma$

- Nous notons $f$ la fonction de $\Sigma$ dans $\mathfrak{a}$ qui $a \mathbf{a}=\left(a_{k}, n_{k}\right)_{k \in \mathbb{Z}}$ associe

$$
f(\mathbf{a})=-\beta\left(a_{0}^{n_{0}}, \eta\right),
$$

ò̀ $\eta=\left(a_{k+1}, n_{k+1}\right)_{k \in \mathbb{N}}$.

- Nous notons $\theta_{f}$ la transformation de $\Sigma \times \mathfrak{a}$ définie pour $(\mathbf{a}, x) \in \Sigma \times \mathfrak{a}$ par

$$
\theta_{f}(\mathbf{a}, x)=(\theta(\mathbf{a}), x+f(\mathbf{a})) .
$$

- Nous notons $(\Sigma \times \mathfrak{a}) /\left\langle\theta_{f}\right\rangle$ l'ensemble des orbites dans $\Sigma \times \mathfrak{a}$ de l'action du groupe $\mathbb{Z}$ induite par la transformation $\theta_{f}$.

L'action par translation de $\mathfrak{a}$ sur $\Sigma \times \mathfrak{a}$ commute avec la transformation $\theta_{f}$ si bien que par passage au quotient cette action induit une action $\left(s_{\alpha} / \alpha \in \mathfrak{a}\right)$ sur l'ensemble $(\Sigma \times \mathfrak{a}) /\left\langle\theta_{f}\right\rangle$ définie, pour tout $\alpha \in \mathfrak{a}$ et toute orbite $\left\{\theta_{f}^{n}(\mathbf{a}, x) / n \in\right.$ $\mathbb{Z}\} \in(\Sigma \times \mathfrak{a}) /\left\langle\theta_{f}\right\rangle$, par

$$
s_{\alpha}\left(\left\{\theta_{f}^{n}(\mathbf{a}, x) / n \in \mathbb{Z}\right\}\right)=\left\{\theta_{f}^{n}(\mathbf{a}, x+\alpha) / n \in \mathbb{Z}\right\}
$$

Le système dynamique ainsi obtenu peut être interprété comme le flot spécial multidimensionnel dont la section de Poincaré est $(\Sigma, \theta)$ et la fonction plafond est $f: \Sigma \rightarrow \mathfrak{a}$. Nous allons démontrer la

Proposition 7.9. - Le flot $\left(w_{\Gamma}^{\alpha} / \alpha \in \mathfrak{a}\right)$ en restriction à $\mathcal{W}_{\Gamma}^{0}$ est conjugué au flot $\left(s_{\alpha} / \alpha \in \mathfrak{a}\right) \operatorname{sur}(\Sigma \times \mathfrak{a}) /\left\langle\theta_{f}\right\rangle$. 
Démonstration de la proposition 7.9. - Considérons l'application $\pi: \Sigma \rightarrow$ $\Lambda_{\Gamma}^{0} \times \Lambda_{\Gamma}^{0}$ qui, à une suite $\left(a_{k}, n_{k}\right)_{n \in \mathbb{Z}}$ de $\Sigma$, associe $\left(\eta_{-}, \eta_{+}\right) \in \Lambda_{\Gamma}^{0} \times \Lambda_{\Gamma}^{0}$ défini par

$$
\eta_{-}:=\lim _{k \rightarrow+\infty}\left(a_{-1}^{-n_{-1}} \ldots a_{-k}^{-n_{-k}}\right) \cdot \xi \text { et } \eta_{+}:=\lim _{k \rightarrow+\infty}\left(a_{0}^{n_{0}} \ldots a_{k}^{n_{k}}\right) \cdot \xi
$$

où $\xi$ est un point fixé de $\mathbf{B}_{\Gamma}$. L'image de $\Sigma$ par $\pi$ est l'ensemble

$$
\mathcal{S}:=\left\{\left(\eta_{-}, \eta_{+}\right) \in \mathcal{F} \times \mathcal{F} / \eta_{ \pm}=\left(a_{k, \pm}, n_{k, \pm}\right)_{k \in \mathbb{N}} \quad \text { et } \quad a_{0,+} \neq a_{0,-}^{ \pm 1}\right\}
$$

et, grâce à l'hypothèse (2) de la définition 6.1, nous avons $\mathcal{S} \subset \partial^{2} \mathcal{F}$. Le décalage $\theta$ de $\Sigma$ induit une transformation sur $\mathcal{S}$, encore notée $\theta$, définie pour $\left(\eta_{-}, \eta_{+}\right)=$ $\pi\left(\left(a_{k}, n_{k}\right)_{k \in \mathbb{Z}}\right) \in \mathcal{S}$ par $\theta\left(\eta_{-}, \eta_{+}\right):=a_{0}^{-n_{0}} \cdot\left(\eta_{-}, \eta_{+}\right)$. On obtient ainsi une équivalence d'orbites entre l'action de $\Gamma$ sur $\partial^{2} \Lambda_{\Gamma}^{0}$ (voir la notation 3.4) et celle de la transformation $\theta$ sur $\mathcal{S}$. Ce qui est suffisant; en effet, fixons $\mathbf{a}=$ $\left(a_{k}, n_{k}\right)_{k \in \mathbb{Z}} \in \Sigma$ et posons $\left(\eta_{-}, \eta_{+}\right)=\pi(\mathbf{a}) \in \mathcal{S}$, on a

$$
\begin{aligned}
a_{0}^{-n_{0}} \cdot\left(\eta_{-}, \eta_{+}, f(\mathbf{a})\right) & =\left(a_{0}^{-n_{0}} \cdot \eta_{-}, a_{0}^{-n_{0}} \cdot \eta_{+}, f(\mathbf{a})-\beta\left(a_{0}^{-n_{0}}, \eta_{+}\right)\right) \\
& =\left(a_{0}^{-n_{0}} \cdot \eta_{-}, a_{0}^{-n_{0}} \cdot \eta_{+}, f(\mathbf{a})+\beta\left(a_{0}^{n_{0}}, a_{0}^{-n_{0}} \cdot \eta_{+}\right)\right) \\
& =\left(\theta\left(\eta_{-}, \eta_{+}\right), 0\right) .
\end{aligned}
$$

D'où le résultat.

La conjugaison obtenue ci-dessus est «abstraite » au sens où le système dynamique $(\Sigma, \theta)$ n'est pas défini sur un espace géométrique. Toutefois, les arguments que nous avons employés montrent que $\Sigma$ s'identifie avec l'ensemble « géométrique » $\mathcal{S}$ dont nous fixons la notation ci-dessous.

Notations 7.10. - Nous notons $\mathcal{S}$ l'ensemble des couples de drapeaux $\left(\eta_{-}, \eta_{+}\right) \in \Lambda_{\Gamma}^{0} \times \Lambda_{\Gamma}^{0}$ tels que

$$
a_{0,+} \neq a_{0,-}^{ \pm 1},
$$

où $\eta_{ \pm}=\left(a_{k,+}, n_{k, \pm}\right)_{k \in \mathbb{N}}$. De plus, nous notons encore $\theta$ la transformation sur $\mathcal{S}, f$ la fonction sur $\mathcal{S}$ et $\theta_{f}$ la transformation sur $\mathcal{S}$ obtenues via l'identification entre $\Sigma$ et $\mathcal{S}$.

Remarques. - (1) Grâce à l'hypothèse (2) de la définition 6.1, on a $\mathcal{S} \subset \partial^{2} \mathcal{F}$.

(2) Puisque la fonction $f$ ne dépend de $\left(a_{k}, n_{k}\right)_{k \in \mathbb{Z}}$ que par ses coordonnées positives; via l'identification entre $\Sigma$ et $\mathcal{S}$, la fonction $f$ ne dépend d'un point $\left(\eta_{-}, \eta_{+}\right) \in \mathcal{S}$ que par $\eta_{+}$.

La proposition qui suit donne l'expression de $m_{\Gamma}$ lorsque $\mathcal{W}_{\Gamma}^{0}$ est identifié $\operatorname{avec}(\mathcal{S} \times \mathfrak{a}) /\left\langle\theta_{f}\right\rangle$. 
Proposition 7.11. - La mesure $m$ restreinte à $\mathcal{S} \times \mathfrak{a}$ est $\theta_{f}$ invariante et induit par passage au quotient la mesure de Patterson-Sullivan $m_{\Gamma}$ sur $\mathcal{W}_{\Gamma}^{0}$. En d'autres termes, pour tous $\Phi_{1}, \Phi_{2} \in \mathcal{B}_{\infty}(\mathcal{S} \times \mathfrak{a}, \mathbb{R})$, on a

$$
\int_{\mathcal{S} \times \mathfrak{a}}\left(\sum_{n \in \mathbb{Z}} \Phi_{1} \circ \theta_{f}^{n}\right) \times \Phi_{2} d m=\int_{\mathcal{S} \times \mathfrak{a}}\left(\sum_{\gamma \in \Gamma} \Phi_{1} \circ \gamma\right) \times \Phi_{2} d m
$$

La démonstration de ce résultat découle directement de ce qui précède. (Voir également le paragraphe 1.4 de [4] où des arguments similaires sont employés).

\section{Feuilletage horosphérique stable de $\Gamma$}

Dans [10], sous certaines hypothèses techniques, Y. Guivarc'h et J. Hardy ont développé une méthode permettant d'établir la propriété de mélange pour un flot spécial défini au dessus d'un espace symbolique et dont la fonction plafond est à valeurs dans $\mathbb{R}$. Une étape importante de leur démonstration consiste à étudier un facteur du système dynamique initial.

Dans la situation géométrique qui nous intéresse, le facteur étudié par Y. Guivarc'h et J. Hardy peut s'interpréter comme étant la projection naturelle du système dynamique $\left(\mathcal{S}, \theta_{f}, m\right)$ sur le feuilletage horosphérique stable dont nous rappelons ci-dessous la définition.

Considérons l'espace homogène $\mathcal{H}:=G / M N$. Par analogie avec le rang 1 (Voir par exemple le paragraphe 1.D de [14]), nous appellerons cet espace feuilletage horosphérique stable de $X$.

Le feuilletage horosphérique $\mathcal{H}$ s'identifie avec l'espace produit $\mathcal{F} \times \mathfrak{a}$. En effet, munissons l'espace $\mathcal{F} \times \mathfrak{a}$ de la topologie produit et faisons opérer le groupe $G$ sur $\mathcal{F} \times \mathfrak{a}$ en associant à $g \in G$ et $(\eta, x) \in \mathcal{F} \times \mathfrak{a}$ le point $g$. $(\eta, x):=(g \cdot \eta, x-\beta(g, \eta))$. Le groupe $G$ opère transitivement sur $\mathcal{F} \times \mathfrak{a}$ et le stabilisateur de $\left(\eta_{0}, 0\right)$ sous l'action de $G$ est le sous-groupe fermé $M N$. Nous en déduisons que l'application

$$
G / M N \rightarrow \mathcal{F} \times \mathfrak{a} \quad g M N \mapsto\left(g \cdot \eta_{0},-\beta\left(g, \eta_{0}\right)\right)
$$

définit un homéomorphisme $G$-équivariant qui nous permet d'identifier ces deux espaces.

D'après la remarque qui suit les notations 7.10 , la fonction $f$ ne dépend de $\left(\eta_{-}, \eta_{+}\right) \in \mathcal{S}$ que par $\eta_{+} \in \Lambda_{\Gamma}^{0}$; la projection naturelle de $\mathcal{W}\left(=\partial^{2} \mathcal{F} \times \mathfrak{a}\right)$ sur $\mathcal{H}(=\mathcal{F} \times \mathfrak{a})$ induit donc un facteur de $\left(\mathcal{S}, \theta_{f}\right)$. Nous fixons les notations suivantes :

\section{Notations 8.1}

- Via l'identification entre $\Lambda_{\Gamma}^{0}$ et $\Sigma_{+}$, on note $\theta_{+}$la transformation sur $\Lambda_{\Gamma}^{0}$ induit par le décalage de $\Sigma_{+}$. 
- On note $f_{+}$la fonction définie sur $\Lambda_{\Gamma}^{0}$ pour $\eta \in \Lambda_{\Gamma}^{0}$ par

$$
f_{+}(\eta)=-\beta\left(a_{0}^{n_{0}}, \theta_{+}(\eta)\right) .
$$

- On note $\theta_{+, f_{+}}$la transformation de $\Lambda_{\Gamma}^{0} \times \mathfrak{a}$ définie pour $\eta \in \Lambda_{\Gamma}^{0}$ par

$$
\theta_{+, f_{+}}(\eta, x)=\left(\theta_{+}(\eta), x+f_{+}(\eta)\right) .
$$

- On note $M$ la mesure sur $\Lambda_{\Gamma}^{0} \times \mathfrak{a}$ définie pour $\Phi \in \mathcal{B}_{\infty}\left(\Lambda_{\Gamma}^{0} \times \mathfrak{a}\right)$ par

$$
\int_{\Lambda_{\Gamma}^{0} \times \mathfrak{a}} \Phi d M=\int \Phi(\eta, x) 1_{\mathcal{S}}\left(\eta_{-}, \eta_{+}\right) d m\left(\eta_{-}, \eta_{+}, x\right) .
$$

À l'instar des méthodes employées dans [10], nous allons à présent étudier les propriétés asymptotiques de la transformation $\theta_{+, f_{+}}$en considérant l'opérateur adjoint qui lui est naturellement associé. Pour ce faire, nous introduisons la

\section{Notations 8.2}

- Nous notons $\tilde{P}$ l'opérateur adjoint de $\theta_{+, f_{+}}$dans $L^{2}\left(\Lambda_{\Gamma}^{0} \times \mathfrak{a}, d M\right)$.

- Nous notons $\mathfrak{L}$, l'ensemble des fonctions $\Phi$ définies sur $\Lambda_{\Gamma}^{0} \times \mathfrak{a}$ de la forme $\Phi=\phi \otimes u$, où $\phi$ est une fonction lispchitzienne sur $\Lambda_{\Gamma}$ et $u$ une fonction sur $\mathfrak{a}$ continue à support compact.

La proposition est une conséquence de l'étude des opérateurs de Ruelle effectuée dans [15] conjuguée à un théorème du renouvellement. Plus précisément, nous allons montrer que l'opérateur $\tilde{P}$ induit naturellement une chaîne semimarkovienne qui vérifie les hypothèses du théorème 10.2. Ce dernier théorème est une légère généralisation d'un théorème du renouvellement dû à $\mathrm{M}$. Babillot.

Proposition 8.3. - Avec les notations introduites ci-dessus. Pour toute fonction $\Phi \in \mathfrak{L}$, la fonction

$$
\Lambda_{\Gamma} \times \mathfrak{a} \rightarrow \mathbb{R} \quad \alpha \mapsto\langle\tau(\Gamma), \alpha\rangle^{\frac{r g(G)-1}{2}} \sum_{n=0}^{+\infty} \tilde{P}^{n}(\Phi)(\eta, \alpha)
$$

est bornée. De plus, il existe une constante $c>0$ et un endomorphisme $S$ sur $\mathfrak{a}$ symétrique et défini positif tels que, pour tout $\Phi \in \mathfrak{L}$ et tout $\alpha \in \mathfrak{a}$, on ait :

$$
\begin{aligned}
& t^{\frac{r g(G)-1}{2}} \sum_{n=0}^{+\infty} \tilde{P}^{n}(\Phi)(\eta,-t \tau(\Gamma)-\sqrt{t} \alpha) \rightarrow \\
& c e^{-\frac{\|S(\alpha)\|^{2}\|S(\tau(\Gamma))\|^{2}-\langle S(\tau(\Gamma)), S(\alpha)\rangle^{2}}{\|S(\tau(\Gamma))\|^{2}}} \int_{\Lambda_{\Gamma} \times \mathfrak{a}} \Phi d M
\end{aligned}
$$

lorsque $t \rightarrow+\infty$ uniformément par rapport à $\eta \in \Lambda_{\Gamma}^{0}$.

Pour établir ce résultat, nous avons besoin de rappeler quelques résultats nous avons démontré dans [15]. À cet effet, fixons quelques notations. 
Notations 8.4. - Pour tout $a \in \mathcal{A}$, nous notons $\Lambda_{a}^{0}$ le sous-ensemble de $\Lambda_{\Gamma}^{0}$ formé des drapeaux $\eta=\left(a_{k}, n_{k}\right)_{k \in \mathbb{N}}$ tels que $a_{0} \neq a^{ \pm 1}$. De plus, pour tout $a \in \mathcal{A}$, nous notons $\Lambda_{a}$ l'adhérence de $\Lambda_{a}^{0}$ dans $\Lambda_{\Gamma}$.

Nous utilisons à présent le vocabulaire de [2] rappelé dans la section 10. Dans [15], nous avons démontré le

Lemme 8.5. - Avec les terminologies utilisées dans la section 10.

1. Il existe une unique fonction $h$ lipschitzienne sur $\Lambda_{\Gamma}$ pour laquelle $\int_{\Lambda_{\Gamma}} h d \nu=1$ et telle que, pour tout $\eta \in \Lambda_{\Gamma}$, on ait

$$
h(\eta)=\sum_{(a, n) \in \mathcal{A} \times \mathbb{N}^{*}} e^{-\left\langle\tau(\Gamma), \beta\left(a^{n}, \eta\right)\right.} h\left(a^{n} \cdot \eta\right) 1_{\Lambda_{a}}(\eta) .
$$

2. La fonction $\tilde{Q}: \Lambda_{\Gamma} \times \mathfrak{a} \rightarrow \mathcal{M}\left(\Lambda_{\Gamma} \times \mathfrak{a}\right)$ définie pour $(\eta, x) \in \Lambda_{\Gamma} \times \mathfrak{a}$ par

$$
\tilde{Q}_{(\eta, \alpha)}=\frac{1}{h(\eta)} \sum_{(a, n) \in \mathcal{A} \times \mathbb{N}^{*}} e^{-\left\langle\tau(\Gamma), \beta\left(a^{n}, \eta\right)\right\rangle} h\left(a^{n} \cdot \eta\right) \mathcal{D}_{a^{n} \cdot(\eta, x)}
$$

est une probabilité de transition, semi-markovienne, adaptée, $L\left(\Lambda_{\Gamma}\right)$ fortement ergodique relativement à la mesure $h d \nu$ et admet des moments à tout ordre.

3. Le vecteur moyenne

$$
\int_{\Lambda_{\Gamma}}\left(\int_{\Lambda_{\Gamma} \times \mathfrak{a}} x_{1} d \tilde{Q}_{(\eta, x)}\left(\eta_{1}, x_{1}\right)\right) h(\eta) d \nu(\eta)
$$

de $\tilde{Q}$ est non nul et colinéaire à $\tau(\Gamma)$.

La démonstration des deux premières assertions repose sur des arguments classiques issus du formalisme thermodynamique. Elle s'obtient en étudiant le spectre des opérateurs de transferts induit par le décalage $\theta_{+}$sur l'ensemble $\Sigma_{+}$. La dernière assertion s'appuie sur le fait que le nombre de retours dans une partie compacte de la chaîne $\left(X_{n}\right)_{n \in \mathbb{N}}$ sur $\Lambda_{\Gamma} \times \mathfrak{a}$ associée à $Q$ décroit exponentiellement vite si le point de départ de la chaîne fuit dans une direction autre que le vecteur moyenne. Plus précisément, pour tout cône ouvert $C$ de a ne contenant pas le vecteur moyenne de $\left(X_{n}\right)_{n \in \mathbb{N}}$, le nombre moyen de retour dans une partie compacte de $\mathfrak{a}$ de $\left(X_{n}\right)_{n \in \mathbb{N}}$ issue de $(\eta,-t \alpha)$ décrô̂t exponentiellement vite si $\alpha \in C$. Plus précisément, on raisonne par l'absurde en supposant que le vecteur moyenne $\vec{\pi}$ de $\left(X_{n}\right)_{n \in \mathbb{N}}$ n'est pas colinéaire au vecteur de croissance de $\Gamma$ si bien qu'il existe un cône $C$ tel que $\tau(\Gamma) \in C$ et $\vec{\pi} \notin C$. Ainsi, par définition de $\tau(\Gamma)$, il existe $\epsilon>0$ tel que $e^{-(\|\tau(\Gamma)\|-\epsilon) R} \operatorname{Card}\{\gamma \in \Gamma / \mu(\gamma) \notin C,\|\mu(\gamma)\| \leq$ $R\} \rightarrow 0$ lorsque $R \rightarrow+\infty$. En utilisant la propriété ci-dessus, on montre qu'il existe $\epsilon>0$ tel que $e^{-(\|\tau(\Gamma)\|-\epsilon) R} \operatorname{Card}\{\gamma \in \Gamma / \mu(\gamma) \in C,\|\mu(\gamma)\| \leq R\} \rightarrow 0$ 
lorsque $R \rightarrow+\infty$. Ce qui contredit le fait que l'exposant critique de la mesure $\sum_{\gamma \in \Gamma} \mathcal{D}_{\|\mu(\gamma)\|}$ soit $\|\tau(\Gamma)\|$.

Démonstration de la proposition 8.3. - Conservons les notations introduites dans le lemme qui précède et notons encore $\tilde{Q}$ l'opérateur sur $\mathcal{B}_{\infty}\left(\Lambda_{\Gamma} \times \mathfrak{a}, \mathbb{R}\right)$ naturellement induit par $\tilde{Q}$.

D'après le théorème 10.2 et le lemme 8.5 , pour toute fonction $\phi: \Lambda_{\Gamma} \rightarrow \mathbb{R}$ lipschitzienne et toute fonction $u: \mathfrak{a} \rightarrow \mathbb{R}$ continue à support compact, la fonction

$$
\Lambda_{\Gamma} \times \mathfrak{a} \rightarrow \mathbb{R} \quad \alpha \mapsto\langle\tau(\Gamma), \alpha\rangle^{\frac{r g(G)-1}{2}} \sum_{n=0}^{+\infty} \tilde{Q}^{n}(\phi \otimes u)(\eta, \alpha)
$$

est bornée. De plus, il existe une constante $c>0$ et un endomorphisme $S$ sur $\mathfrak{a}$ symétrique et défini positif tels que, pour toute fonction $\phi: \Lambda_{\Gamma} \rightarrow \mathbb{R}$ lipschitzienne et toute fonction $u: \mathfrak{a} \rightarrow \mathbb{R}$ continue à support compact, on ait :

$$
\begin{aligned}
t^{\frac{r g(G)-1}{2}} & \sum_{n=0}^{+\infty} \tilde{Q}^{n}(\phi \otimes u)(\eta,-t \tau(\Gamma)-\sqrt{t} \alpha) \\
\rightarrow & c e^{-\frac{\|S(\alpha)\|^{2}\|S(\tau(\Gamma))\|^{2}-\langle S(\tau(\Gamma)), S(\alpha)\rangle^{2}}{\|S(\tau(\Gamma))\|^{2}}} \int_{\Lambda_{\Gamma} \times \mathfrak{a}} \phi \otimes u(\eta, x) h(\eta) d \nu(\eta) \otimes d x .
\end{aligned}
$$

lorsque $t \rightarrow+\infty$. Ainsi, le borélien $\Lambda_{\Gamma}^{0}$ étant de $\nu$-mesure nulle, il nous suffit de montrer que, pour tout $\Phi \in \mathfrak{L}$ et pour $M$-presque tout $(\eta, x) \in \Lambda_{\Gamma}^{0} \times \mathfrak{a}$, on a

$$
\tilde{P}(\Phi)(\eta, x)=\int_{\Lambda_{\Gamma} \times \mathfrak{a}} \Phi\left(\eta_{1}, x_{1}\right) d \tilde{Q}_{(\eta, x)}\left(\eta_{1}, x_{1}\right)
$$

Pour ce faire, notons que, pour tout borélien $A$ de $\Lambda_{\Gamma}^{0} \times \mathfrak{a}$, on a

$$
M(A)=\int_{\Lambda_{\Gamma}^{0} \times \mathfrak{a}} 1_{A}(\eta, x) \tilde{h}(\eta) d \nu(\eta) \otimes d x
$$

où $\tilde{h}$ est la fonction définie sur $\Lambda_{\Gamma}^{0}$ pour $\eta \in \Lambda_{\Gamma}^{0}$ par

$$
\tilde{h}(\eta)=\int_{\Lambda_{\Gamma}} 1_{\mathcal{S}}\left(\eta, \eta_{+}\right) \frac{d \nu\left(\eta_{+}\right)}{e^{-\left[\eta, \eta_{+}\right]_{\tau(\Gamma)}}} .
$$

Ainsi, pour tout $\Phi \in \mathfrak{L}$ et $M$-presque tout $(\eta, x) \in \Lambda_{\Gamma}^{0} \times \mathfrak{a}$,

$$
\tilde{P}(\Phi)(\eta, x)=\frac{1}{\tilde{h}(\eta)} \sum_{(a, n) \in \mathcal{A} \times \mathbb{N}^{*}} \tilde{h}\left(a^{n} \cdot \eta\right) e^{-\left\langle\tau(\Gamma), \beta\left(a^{n}, \eta\right)\right\rangle} \Phi\left(a^{n} \cdot(\eta, x)\right) 1_{\Lambda_{a}^{0}}(\eta) .
$$

D'autre part, observons que, pour tout $\eta \in \Lambda_{\Gamma}^{0}$

$$
\tilde{h}(\eta)=\sum_{(a, n) \in \mathcal{A} \times \mathbb{N}^{*}} e^{-\left\langle\tau(\Gamma), \beta\left(a^{n}, \eta\right)\right.} \tilde{h}\left(a^{n} \cdot \eta\right) 1_{\Lambda_{a}^{0}}(\eta) .
$$


Ainsi, le produit de Gromov étant $C^{\infty}$, la fonction $\tilde{h}$ est lipschitzienne sur $\Lambda_{\Gamma}^{0}$. Ceci montre que le prolongement par continuité de $\tilde{h}$ sur $\Lambda_{\Gamma}$ existe et coïncide avec $h$. D'où le résultat.

\section{Propriétés de mélange des groupes de Ping-Pong}

Nous allons démontrer le :

ThÉorème 9.1. - Pour tous $\psi_{1}, \psi_{2} \in C_{c}\left(\mathcal{W}_{\Gamma}, \mathbb{R}\right)$, la fonction

$$
\mathfrak{a} \rightarrow \mathbb{R} \quad \alpha \mapsto\langle\tau(\Gamma), \alpha\rangle^{\frac{r g(G)-1}{2}} m_{\Gamma}\left(\psi_{1} \times \psi_{2} \circ w_{\Gamma}^{\alpha}\right)
$$

est bornée. De plus, il existe une constante $c>0$ et un endomorphisme $S$ symétrique et défini positif sur $\mathfrak{a}$ tels que, pour tout $\alpha \in \mathfrak{a}$ et tous $\psi_{1}, \psi_{2} \in$ $C_{c}\left(\mathcal{W}_{\Gamma}, \mathbb{R}\right)$, on ait :

$$
\begin{aligned}
& t^{\frac{r g(G)-1}{2}} m_{\Gamma}\left(\psi_{1} \times \psi_{2} \circ w_{\Gamma}^{t \tau(\Gamma)+\sqrt{t} \alpha}\right) \rightarrow \\
& c e^{-\frac{\|S(\tau(\Gamma))\|^{2}\|S(\alpha)\|^{2}-\langle S(\tau(\Gamma)), S(\alpha)\rangle^{2}}{\|S(\tau(\Gamma))\|^{2}}} m_{\Gamma}\left(\psi_{1}\right) m_{\Gamma}\left(\psi_{2}\right)
\end{aligned}
$$

lorsque $t \rightarrow+\infty$.

Remarques. - (1) Lorsque le rang de $G$ vaut 1 , ce résultat a été démontré dans [8] (Théorème VI.2) et dans ce cas la mesure de Patterson-Sullivan est finie. En fait, la mesure de Patterson-Sullivan d'un groupe de Ping-Pong est finie si et seulement si $r g(G)=1$.

(2) Ce résultat fait apparaître une différence frappante entre les groupes de Ping-Pong et les réseaux. En effet, la mesure de Patterson-Sullivan d'un réseau est finie et le flot des chambres de Weyl associé à un réseau est mélangeant relativement à cette mesure; le terme « polynomial» $t^{\frac{r g(G)-1}{2}}$ n'apparaît pas dans ce cas.

Démonstration du théorème 9.1. - Afin d'alléger le texte, nous introduisons la notation suivante

Notation 9.2. - On fixe une constante $c>0$ et un endomorphisme $S$ symétrique et défini positif sur a comme dans la proposition 8.3. On note $F$ la fonction définie sur a pour $\alpha \in \mathfrak{a} \operatorname{par} F(\alpha):=c e^{-\frac{\|S(\alpha)\|^{2}\|S(\tau(\Gamma))\|^{2}-\langle S(\tau(\Gamma)), S(\alpha)\rangle^{2}}{\|S(\tau(\Gamma))\|^{2}}}$. 
Il est suffisant de montrer que, pour tout $\Psi_{1}, \Psi_{2} \in C_{c}(\mathcal{W}, \mathbb{R})$, la fonction

$$
\mathfrak{a} \rightarrow \mathbb{R} \quad \alpha \mapsto\langle\tau(\Gamma), \alpha\rangle^{\frac{r g(G)-1}{2}} \sum_{\gamma \in \Gamma} m\left(\Psi_{1} \circ \gamma \times \Psi_{2} \circ w_{\alpha}\right)
$$

est bornée et qu'il existe une constante $c>0$ et un endomorphisme $S$ sur a symétrique et défini positif tel que, pour tout $\alpha \in \mathfrak{a}$ et tous $\Psi_{1}, \Psi_{2} \in C_{c}(\mathcal{W}, \mathbb{R})$, on a

$$
\begin{gathered}
t^{\frac{r g(G)-1}{2}} \sum_{\gamma \in \Gamma} m\left(\Psi_{1} \circ \gamma \times \Psi_{2} \circ w_{t \tau(\Gamma)+\sqrt{t} \alpha}\right) \\
\rightarrow F(\alpha) m\left(\Psi_{1}\right) m\left(\Psi_{2}\right)
\end{gathered}
$$

lorsque $t \rightarrow+\infty$.

On décompose la démonstration de ce théorème en deux étapes. On commence par montrer que les affirmations qui précèdent sont vraies pour une classe de fonction test puis on étendra ces propriétés à la classe de fonctions voulue.

Introduisons les

Notations 9.3. - On note $L\left(\Lambda_{\Gamma}\right)$ l'espace des fonctions lipschitziennes sur $\Lambda_{\Gamma}$.

On note $\mathfrak{F}$ l'ensemble formé des fonctions $\Phi \in \mathcal{B}_{\infty}(\mathcal{W}, \mathbb{C})$ pour lesquelles il existe $n \in \mathbb{N}, \phi \in L\left(\Lambda_{\Gamma}\right)$ et $u \in C_{c}(\mathfrak{a}, \mathbb{R})$ tels que, pour tout $\left(\eta_{-}, \eta_{+}, \alpha\right) \in \mathcal{W}$, on ait

$$
\Phi\left(\eta_{-}, \eta_{+}, \alpha\right)=\phi\left(\eta_{+}\right) u\left(\alpha+S_{n} f\left(\eta_{-}, \eta_{+}\right)\right) 1_{\mathcal{S}}\left(\eta_{-}, \eta_{+}\right)
$$

Étape 1. Montrons que, pour tout $\Phi^{(1)}, \Phi^{(2)} \in \mathfrak{F}$, la fonction

$$
\mathfrak{a} \rightarrow \mathbb{R} \quad \alpha \mapsto\langle\tau(\Gamma), \alpha\rangle^{\frac{r g(G)-1}{2}} \sum_{\gamma \in \Gamma} \int_{\mathcal{W}} \Phi^{(1)} \circ \gamma \times \Phi^{(2)} \circ w_{\alpha} d m
$$

est bornée et, pour tout $\alpha \in \mathfrak{a}$, on a :

$$
t^{\frac{r g(G)-1}{2}} \sum_{\gamma \in \Gamma} \int_{\mathcal{W}} \Phi^{(1)} \circ \gamma \times \Phi^{(2)} \circ w_{t \tau(\Gamma)+\sqrt{t} \alpha} \rightarrow F(\alpha) \int_{\mathcal{W}} \Phi^{(1)} d m \int_{\mathcal{W}} \Phi^{(2)} d m
$$

lorsque $t \rightarrow+\infty$.

Nous choisissons $n_{1}, n_{2} \in \mathbb{N}$, deux fonctions $\phi^{(1)}, \phi^{(2)} \in L\left(\Lambda_{\Gamma}\right)$ et $\operatorname{deux}$ fonctions $u_{1}, u_{2} \in C_{c}(\mathfrak{a}, \mathbb{R})$ telles que, pour tout $\left(\eta_{-}, \eta_{+}, x\right) \in \mathcal{W}$, on ait

$$
\Phi^{(1)}\left(\eta_{-}, \eta_{+}, x\right)=\phi^{(1)}\left(\eta_{+}\right) u_{1}\left(x+S_{n_{1}} f_{+}\left(\eta_{+}\right)\right) 1_{\mathcal{S}}\left(\eta_{-}, \eta_{+}\right)
$$

et

$$
\Phi^{(2)}\left(\eta_{-}, \eta_{+}, x\right)=\phi^{(2)}\left(\eta_{+}\right) u_{2}\left(x+S_{n_{2}} f_{+}\left(\eta_{+}\right)\right) 1_{\mathcal{S}}\left(\eta_{-}, \eta_{+}\right) .
$$


Pour tout $\alpha \in \mathfrak{a}$, nous posons

$$
I(\alpha):=\sum_{\gamma \in \Gamma} \int_{\mathcal{W}} \Phi^{(1)} \circ \gamma \times \Phi^{(2)} \circ w_{\alpha} d m .
$$

D'après la proposition 8.3 , pour tout $\alpha \in \mathfrak{a}$, on a

$$
I(\alpha)=\sum_{n \in \mathbb{Z}} \int_{\mathcal{S} \times \mathfrak{a}} \Phi^{(1)} \circ \theta_{f}^{n}(\eta, x) \times \Phi^{(2)}\left(\eta_{+}, x-\alpha\right) d m\left(\eta_{-}, \eta_{+}, x\right) .
$$

Ainsi, on obtient $I(\alpha)=I^{+}(\alpha)+I^{-}(\alpha)$ avec

$$
I^{+}(\alpha)=\sum_{n=0}^{+\infty} \int_{\mathcal{S} \times \mathfrak{a}} \Phi^{(1)} \circ \theta_{f}^{n}(\eta, x) \times \Phi^{(2)}\left(\eta_{+}, x-\alpha\right) d m\left(\eta_{-}, \eta_{+}, x\right)
$$

et

$$
I^{-}(\alpha)=\sum_{n=1}^{+\infty} \int_{\mathcal{S} \times \mathfrak{a}} \Phi^{(1)} \circ \theta_{f}^{-n}(\eta, x) \times \Phi^{(2)}\left(\eta_{+}, x-\alpha\right) d m\left(\eta_{-}, \eta_{+}, x\right) .
$$

La mesure $m$ étant $\theta_{f}$ invariante, on a

$$
I^{-}(\alpha)=\sum_{n=1}^{+\infty} \int_{\mathcal{S} \times \mathfrak{a}} \Phi^{(1)}(\eta, x) \times \Phi^{(2)} \circ \theta_{f}^{n}\left(\eta_{+}, x-\alpha\right) d m\left(\eta_{-}, \eta_{+}, x\right) .
$$

Décrivons le comportement asymptotique de $\alpha \mapsto I^{+}(\alpha)$ et $\alpha \mapsto I^{-}(\alpha)$. À cet effet, notons $\Psi^{(1)}$ et $\Psi^{(2)}$ les fonctions définies sur $\Lambda_{\Gamma}^{0}$ par

$$
\Psi^{(1)}(\eta, x)=\phi^{(1)}(\eta) u_{1}\left(x+S_{n_{1}} f_{+}\left(\eta_{+}\right)\right)
$$

et

$$
\Psi^{(2)}\left(\eta_{+}, x\right)=\phi^{(2)}\left(\eta_{+}\right) u_{2}\left(x+S_{n_{2}} f_{+}\left(\eta_{+}\right)\right) .
$$

Par définition de $M$, nous avons

$$
\begin{aligned}
I^{+}(\alpha)= & \sum_{n=0}^{+\infty} \int_{\Lambda_{\Gamma}^{0} \times \mathfrak{a}} \Psi^{(1)} \circ \theta_{+, f_{+}}^{n}\left(\eta_{+}, x+S_{n_{1}} f\left(\eta_{+}\right)\right) \\
& \times \Psi^{(2)}\left(\eta_{+}, x-\alpha+S_{n_{2}} f\left(\eta_{+}\right)\right) d M\left(\eta_{+}, x\right) \\
= & \int_{\Lambda_{\Gamma}^{0} \times \mathfrak{a}} \Psi^{(1)}\left(\eta_{+}, x+S_{n_{1}} f\left(\eta_{+}\right)-S_{n_{2}} f\left(\eta_{+}\right)\right) \\
& \times\left(\sum_{n=0}^{+\infty} \tilde{P}^{n}\left(\Psi^{(2)}\right)\left(\eta_{+}, x-\alpha\right)\right) d M\left(\eta_{+}, x\right)
\end{aligned}
$$

Ces égalités conjuguées à la proposition 8.3 montrent que

$$
\mathfrak{a} \rightarrow \mathbb{R}, \alpha \mapsto\langle\tau(\Gamma), \alpha\rangle^{\frac{r g(G)-1}{2}} \times I^{+}(\alpha)
$$

TOME $137-2009-\mathrm{N}^{\mathrm{O}} 3$ 
est bornée et que, pour tous $\alpha, x \in \mathfrak{a}$, on a

$$
t^{\frac{r g(G)-1}{2}} \sum_{n=0}^{+\infty} \tilde{P}^{n}\left(\Psi^{(2)}\right)\left(\eta_{+}, x-t \tau(\Gamma)-\sqrt{t} \alpha\right) \rightarrow F(\alpha) \int_{\Lambda_{\Gamma} \times \mathfrak{a}} \Psi^{(2)} d M
$$

lorsque $t \rightarrow+\infty$ et uniformément par rapport à $\eta_{+} \in \Lambda_{\Gamma}$. Le théorème de convergence dominée de Lebesgue assure que, pour tout $\alpha \in \mathfrak{a}$, on a

$$
t^{\frac{r g(G)-1}{2}} \times I^{+}(t \tau(\Gamma)+\sqrt{t} \alpha) \rightarrow F(\alpha) \int_{\mathcal{W}} \Phi^{(1)} d m \int_{\mathcal{W}} \Phi^{(2)} d M
$$

lorsque $t \rightarrow+\infty \operatorname{car} \int_{\Lambda_{\Gamma} \times \mathfrak{a}} \Psi^{(2)} d M=\int_{\mathcal{W}} \Phi^{(2)} d m$

Ainsi, on conclut en décrivant le comportement asymptotique de $\alpha \mapsto I^{-}(\alpha)$ par les mêmes arguments. D'où l'étape 1.

Étape 2. Conclusion. - Nous utiliserons la notation suivante : Pour tout $\alpha \in \mathfrak{a}$ et tous $\Psi_{-}, \Psi_{+} \in \mathcal{B}_{\infty}(\mathcal{W}, \mathbb{C})$, nous posons

$$
J\left(\alpha, \Psi_{-}, \Psi_{+}\right)=\langle\tau(\Gamma), \alpha\rangle^{\frac{r g(G)-1}{2}} \sum_{\gamma \in \Gamma} \int_{\mathcal{W}} \Psi_{-} \circ \gamma \times \Psi_{+} \circ w_{\alpha} d m .
$$

Nous utiliserons également le lemme suivant qui permet de restreindre notre étude aux cas des fonctions continues sur $\mathcal{W}$ et dont le support est contenu dans $\mathcal{S} \times \mathfrak{a}$.

LEMME 9.4. - L'ensemble $\mathcal{S}$ est ouvert dans $\partial^{2} \Lambda_{\Gamma}$ et la famille $(\gamma \cdot \mathcal{S} / \gamma \in \Gamma)$ recouvre $\partial^{2} \Lambda_{\Gamma}$.

D'après ce lemme, on peut supposer que le support des fonctions $\Psi_{-}$et $\Psi_{+}$ est contenu dans $\mathcal{S} \times \mathfrak{a}$; en effet, par le théorème de partition de l'unité et le lemme 9.4, toute fonction continue $\Psi: \partial^{2} \Lambda_{\Gamma} \times \mathfrak{a} \rightarrow \mathbb{R}$ à support compact s'écrit $\sum_{\gamma \in \Gamma} \Psi_{\gamma} \circ \gamma$ où $\left(\Psi_{\gamma} / \gamma \in \Gamma\right)$ est une famille de fonctions continues sur $\partial^{2} \Lambda_{\Gamma} \times \mathfrak{a}$ dont le support est contenu dans $\mathcal{S} \times \mathfrak{a}$.

Les méthodes employées par Y. Guivarc'h et J. Hardy dans [10] reposent sur une approximation uniforme qui ne peut s'appliquer directement à notre situation lorsque $\operatorname{rg}(G) \geq 2$. En effet, la présence de la fonction non constante $\mathbb{R} \rightarrow \mathbb{R}, t \mapsto t^{\frac{r g(G)-1}{2}}$ fait « exploser » toute approximation uniforme.

Dans le lemme qui suit, nous précisons un résultat d'approximation des fonctions de $C_{c}(\overline{\mathcal{S}} \times \mathfrak{a}, \mathbb{C})$ par les éléments de $\mathfrak{F}$ qui permettra de conclure.

Lemme 9.5. - Pour tout $\epsilon>0$ et tout $\Psi \in C_{c}(\overline{\mathcal{S}} \times \mathfrak{a}, \mathbb{C})$, il existe $n \in \mathbb{N}$ et $\Phi^{(1)}, \Phi^{(2)} \in \mathfrak{F}$ tels que, pour tout $\left(\eta_{-}, \eta_{+}, x\right) \in \mathcal{S} \times \mathfrak{a}$, on ait

$$
\left|\Psi \circ \theta_{f}^{n}\left(\eta_{-}, \eta_{+}, x\right)-\Phi^{(1)}\left(\eta_{-}, \eta_{+}, x\right)\right|<\epsilon \Phi^{(2)}\left(\eta_{-}, \eta_{+}, x\right)
$$


et

$$
\int_{\mathcal{W}}\left|\Phi^{(2)}\right| d m \leq \operatorname{Vol}\left(\mathbb{B}_{\mathfrak{a}}(0, R+2)\right)+1,
$$

où $\operatorname{Vol}\left(\mathbb{B}_{\mathfrak{a}}(0, R)\right)$ désigne le volume de $\mathbb{B}_{\mathfrak{a}}(0, R)$ par rapport à la mesure de Lebesgue.

Fixons $\epsilon>0$. À l'aide du lemme 9.5 appliqué à la fonction $\Psi_{+}$(resp. $\Psi_{-}$), nous fixons $\Phi_{+}^{(1)}$ et $\Phi_{+}^{(2)} \in \mathfrak{F}\left(\right.$ resp. $\Phi_{-}^{(1)}$ et $\Phi_{-}^{(2)}$ ) vérifiant les inégalités (2) et (3).

Quitte à composer la restriction de $\Psi_{-}\left(\right.$resp $\left.\Psi_{+}\right)$à $\mathcal{S} \times \mathfrak{a}$ par une puissance suffisamment grande de $\theta_{f}$, nous supposerons que, pour tout $\left(\eta_{-}, \eta_{+}, x\right) \in \mathcal{S} \times \mathfrak{a}$, on a

$$
\left|\Psi_{-}\left(\eta_{-}, \eta_{+}, x\right)-\Phi_{-}^{(1)}\left(\eta_{-}, \eta_{+}, x\right)\right|<\epsilon \Phi_{-}^{(2)}\left(\eta_{-}, \eta_{+}, x\right)
$$

(resp. $\left.\left|\Psi_{+}\left(\eta_{-}, \eta_{+}, x\right)-\Phi_{+}^{(1)}\left(\eta_{-}, \eta_{+} r, x\right)\right|<\epsilon \Phi_{+}^{(2)}\left(\eta_{-}, \eta_{+}, x\right)\right)$.

Montrons que la fonction $\mathfrak{a} \rightarrow \mathbb{R}, \alpha \mapsto I\left(\alpha, \Psi_{-}, \Psi_{+}\right)$est bornée. À cet effet, notons que, pour tout $\alpha \in \mathfrak{a}$, on a

$$
I\left(\alpha,\left|\Psi_{-}-\Phi_{-}^{(1)}\right|, \Phi_{+}^{(2)}\right) \leq \epsilon I\left(\alpha, \Phi_{-}^{(2)}, \Phi_{+}^{(2)}\right) .
$$

Ainsi, en utilisant l'étape 1 , nous pouvons affirmer que la fonction $\mathfrak{a} \rightarrow \mathbb{R}$, $\alpha \mapsto I\left(\alpha, \Psi_{-}, \Phi_{+}^{(2)}\right)$ est bornée. De même, pour tout $\alpha \in \mathfrak{a}$, on a

$$
I\left(\alpha, \Psi_{-},\left|\Psi_{+}-\Phi_{+}^{(1)}\right|\right) \leq \epsilon I\left(\alpha, \Psi_{-}, \Phi_{+}^{(2)}\right),
$$

si bien que la fonction $\mathfrak{a} \rightarrow \mathbb{R}, \alpha \mapsto I\left(\alpha, \Psi_{-}, \Psi_{+}\right)$est bornée.

Les mêmes arguments montrent également que, pour tout $\alpha \in \mathfrak{a}$, on a

$$
I\left(t \tau(\Gamma)+\sqrt{t} \alpha, \Psi_{-}, \Psi_{+}\right) \rightarrow F(\alpha) \int_{\mathcal{W}} \Psi_{-} d m \int_{\mathcal{W}} \Psi_{+} d m
$$

lorsque $t \rightarrow+\infty$. Ce qui est suffisant.

\section{Appendice. Un théorème du renouvellement}

Dans cette section, on se propose de démontrer un théorème du renouvellement pour une chaîne semi-markovienne transiente $\left(X_{n}\right)_{n \in \mathbb{N}}=\left(\xi_{n}, \alpha_{n}\right)_{n \in \mathbb{N}}$ sur $\Xi \times \mathbb{R}^{r}$, avec $r \geq 1$, où $\Xi$ désigne un espace métrique compact. Notons $\tilde{Q}$ la probabilité de transition de $\left(X_{n}\right)_{n \in \mathbb{N}}$ et $U$ le noyau potentiel défini par

$$
U:=\sum_{n=0}^{+\infty} \tilde{Q}^{n}
$$

Pour tous boréliens $B \subset \Xi$ et $C \subset \mathbb{R}^{r}$, la quantité $U_{(\xi, \alpha)}(B \times C)$ mesure le nombre moyen de visites de la chaîne semi-markovienne dans $B \times C$ partant de $(\xi, \alpha)$. Le principal résultat de cette section est une estimation asymptotique 
de $U_{(\xi, \alpha)}(B \times C)$ lorsque $C$ est un compact de $\mathbb{R}^{r}$ et que $\alpha$ se trouve « loin » de $C$, dans un sens que nous précisons.

10.1. Énoncé d'un théorème du renouvellement. - Dans cette section, on énonce un théorème du renouvellement relatif à la classe des probabilités de transitions semi-markoviennes introduites par M. Babillot et dont on rappelle ici la définition. On rappelle la

DÉFInItion 10.1. - Soit $\Xi$ un espace topologique de tribu borélienne $\mathcal{X}$. Une probabilité de transition borélienne $\tilde{Q}$ sur $\Xi \times \mathbb{R}^{r}$, avec $r \geq 1$, est dite semimarkovienne si, pour toute fonction $F$ borélienne bornée sur $\Xi \times \mathbb{R}^{r}$ et tout vecteur $\alpha \in \mathbb{R}^{r}$, on a

$$
\int_{\Xi \times \mathbb{R}^{r}} F\left(\xi_{1}, \alpha_{1}\right) d \tilde{Q}_{(\xi, \alpha)}\left(\xi_{1}, \alpha_{1}\right)=\int_{\Xi \times \mathbb{R}^{r}} F\left(\xi_{1}, \alpha_{1}+\alpha\right) d \tilde{Q}_{(\xi, 0)}\left(\xi_{1}, \alpha_{1}\right) .
$$

10.1.0.3. Notations. - On note $\langle.,$.$\rangle le produit scalaire usuel de \mathbb{R}^{r}$.

Soit $\tilde{Q}$ une probabilité de transition borélienne et semi-markovienne sur $\Xi \times$ $\mathbb{R}^{r}$. On note $\left(\left(\Xi \times \mathbb{R}^{r}\right)^{\otimes \mathbb{N}}, \mathcal{B}\left(\left(\Xi \times \mathbb{R}^{r}\right)^{\otimes \mathbb{N}}\right),\left(\mathbb{P}_{(\xi, \alpha)} /(\xi, \alpha) \in \Xi \times \mathbb{R}^{r}\right),\left(X_{n}\right)_{n \in \mathbb{N}}\right)$ la chaîne de Markov canonique associée à la probabilité de transition $Q$; pour tout $(\xi, \alpha) \in \Xi \times \mathbb{R}^{r}$, on notera aussi $\mathbb{E}_{(\xi, \alpha)}$ l'espérance associée à $\mathbb{P}_{(\xi, \alpha)}$.

On note $\mathcal{B}_{\infty}(\Xi, \mathbb{C})$ l'espace des fonctions boréliennes et bornées sur $\Xi$ à valeurs dans $\mathbb{C}$ muni de la norme uniforme. De plus, pour tout $\lambda \in \mathbb{R}^{r}$, on note $Q_{\lambda}$ l'opérateur de $\mathcal{B}_{\infty}(\Xi, \mathbb{C})$, dit de Fourier, défini pour $\psi \in \mathcal{B}_{\infty}(\Xi, \mathbb{C})$ par

$$
Q_{\lambda}(\psi)(\xi)=\int_{\Xi \times \mathbb{R}^{r}} \psi\left(\xi_{1}\right) e^{i\left\langle\lambda, \alpha_{1}\right\rangle} d \tilde{Q}_{(\xi, 0)}\left(\xi_{1}, \alpha_{1}\right) .
$$

On considère l'espace $L(\Xi)$ des fonctions lipschitziennes sur $\Xi$ à valeurs dans $\mathbb{C}$ muni de la norme $\|\cdot\|_{L(\Xi)}$ définie pour $\phi \in L(\Xi)$ par $\|\phi\|_{L(\Xi)}=\|\phi\|_{\infty}+[\phi]$ où $[\phi]$ désigne la constante de Lipschitz de $\phi$. De plus, on note $\mathfrak{L}$ l'espace vectoriel engendré par les fonctions $\Psi: \Xi \times \mathbb{R}^{r} \rightarrow \mathbb{C}$ de la forme $\Psi=\psi \otimes u$ où $\psi$ est une fonction lipschitzienne sur $\Xi$ et $u$ est une fonction continue à support compact sur $\mathbb{R}^{r}$.

10.1.0.4. Hypothèses. - On introduit les hypothèses suivantes :

- R0. L'opérateur $Q_{0}$ agit continûment sur $L(\Xi)$.

- R1. ( $L(\Xi)$-forte ergodicité.) Il existe une mesure de probabilité borélienne $\nu$ définie sur $\Xi$ et un endomorphisme $R$ de $L(\Xi)$ de rayon spectral $<1$ tels que, pour tout $n \geq 1$ et $\phi \in L(\Xi)$, on ait

$$
Q_{0}^{n}(\phi)=1_{\Xi} \int_{\Xi} \phi d \nu+R^{n}(\phi)
$$

- R2. (Adaptation) Le rayon spectral sur $L(\Xi)$ de l'opérateur $Q_{\lambda}$ est strictement inférieur à 1 dès que $\lambda$ est un vecteur non nul de $\mathbb{R}^{r}$. 
- R3. (Moments de tout ordre) Pour tout $p \geq 1$, la fonction $\xi \mapsto$ $\int_{\Omega}\left\|\alpha_{1}\right\|^{p} d \tilde{Q}_{(\xi, 0)}\left(\xi_{1}, \alpha_{1}\right)$ est finie et lipschtizienne sur $\Xi$.

- R4. (Transience) Le vecteur

$$
\vec{M}:=\int_{\Xi} \int_{\Xi \times \mathbb{R}^{r}} \alpha_{1} d \tilde{Q}_{(\xi, 0)}\left(\xi_{1}, \alpha_{1}\right) d \nu(\xi)
$$

est non nul.

10.1.0.5. Énoncé du théorème du renouvellement. — Nous avons le

ThÉORÈme 10.2. - Avec les notations introduites ci-dessus. Si la probabilité de transition $\tilde{Q}$ vérifie les hypothèses $\mathrm{R} 0, \ldots, \mathrm{R} 4$ alors, pour tout $\Phi \in \mathfrak{L}$, la fonction

$$
\Xi \times \mathbb{R}^{r} \rightarrow \mathbb{R} \quad(\xi, \alpha) \mapsto\langle\vec{M}, \alpha\rangle^{\frac{r-1}{2}} \mathbb{E}_{(\xi, \alpha)}\left(\sum_{n=0}^{+\infty} \Phi\left(X_{n}\right)\right)
$$

est bornée; de plus, il existe une matrice $S$ carrée d'ordre $r$, symétrique et définie positive et $c>0$ tels que, pour tout $\Psi \in \mathfrak{L}$ et $f \in \mathbb{R}^{r}$, on ait

$$
\begin{aligned}
& t^{\frac{r-1}{2}} \mathbb{E}_{(\xi,-t \vec{M}-\sqrt{t} f)}\left(\sum_{n=0}^{+\infty} \Psi\left(X_{n}\right)\right) \rightarrow \\
& c e^{-\frac{\|S(f)\|^{2}\|S(\vec{M})\|^{2}-\langle S(f), S(\vec{M})\rangle^{2}}{2\|S(\vec{M})\|^{2}}} \int_{\Xi \times \mathbb{R}^{r}} \Psi(\xi, \alpha) d \nu(\xi) \otimes d \alpha
\end{aligned}
$$

lorsque $t \rightarrow+\infty$, uniformément sur $\Xi$.

10.2. Démonstration du théorème du renouvellement. - La convergence obtenu dans le théorème 10.2 est due à $\mathrm{M}$. Babillot lorsque $f=0$. Les méthodes employées dans [2] s'adaptent à notre situation et nous nous contentons de rappeler les outils nécessaires et de préciser les grandes lignes de la démonstration.

10.2.0.6. La classe des fonctions $\mathcal{H}_{\Xi}$ - - À l'instar des méthodes employées dans [2], nous introduisons la classe $\mathcal{H}$ des fonctions définies sur $\mathbb{R}^{r}$ à valeurs dans $\mathbb{C}$ dont la transformée de Fourier est de classe $C^{N}$ à support compact sur $\mathbb{R}^{r}$, avec $N \geq r$. Nous avons le

LEMme 10.3. - (Lemme $2.4[3])$ Soit $\left(\nu_{T}^{\xi} / \xi \in \Xi, T \in \mathbb{R}^{+}\right)$une famille de mesures de Radon sur $\mathbb{R}^{r}$. S'il existe une mesure $\nu$ pour laquelle les fonctions de $\mathcal{H}$ sont $\nu$-intégrables et telle que, pour tout $f \in \mathcal{H}$, la fonction $\Xi \times \mathbb{R}^{r} \rightarrow \mathbb{R}$, $(\xi, x) \mapsto \nu_{T}^{\xi}(f)$ converge vers $\nu(f)$ uniformément pour $\xi \in \Xi$ lorsque $T \rightarrow+\infty$ alors les mêmes conclusions sont vraies pour les fonctions continues à support compact sur $\mathbb{R}^{r}$. 
Ainsi, en notant $\mathcal{H}_{\Xi}$ l'espace des fonctions de la forme $\Phi=\phi \otimes u$, avec $\phi \in L(\Xi)$ et $\phi \in \mathcal{H}$, nous avons le

Corollaire 10.4. - Soient $c>0$ et $S$ une matrice carrée d'ordre r, symétrique et définie positive. Si, pour tout $\Psi \in \mathcal{H}_{\Xi}$ et $f \in \mathbb{R}^{r}$, la fonction $\mathbb{R}^{+} \rightarrow \mathbb{R}$, $t \mapsto t^{\frac{r-1}{2}} \mathbb{E}_{(\xi,-t \vec{M}-\sqrt{t} f)}\left(\sum_{n=0}^{+\infty} \Psi\left(X_{n}\right)\right)$ converge uniformément sur $\Xi$ lorsque $t \rightarrow+\infty$ vers

$$
c e^{-\frac{\|S(f)\|^{2}\|S(\vec{M})\|^{2}-\langle S(f), S(\vec{M})\rangle^{2}}{2\|S(\vec{M})\|^{2}}} \int_{\Xi \times \mathbb{R}^{r}} \Psi(\xi, x) d \nu(\xi) \otimes d x
$$

alors cette convergence se produit également pour tout $\Psi \in \mathfrak{L}$ et tout $f \in \mathbb{R}^{r}$.

Nous avons également le

Corollaire 10.5. - Si on suppose que, pour tout $\Psi \in \mathcal{H}_{\Xi}$, la fonction

$$
\mathbb{R}^{r} \rightarrow \mathbb{R} \quad x \mapsto\langle\vec{M}, x\rangle^{\frac{r-1}{2}} \mathbb{E}_{(\xi, x)}\left(\sum_{n=0}^{+\infty} \Psi\left(X_{n}\right)\right)
$$

est bornée, il en est de même pour toute fonction $\Psi \in \mathfrak{L}$.

Démonstration du corollaire 10.5. - Considérons une fonction $\Phi \in \mathfrak{L}$. Pour tout $a \in \mathbb{R}$, nous notons $g_{a}$ la fonction définie sur $\Xi \times \mathfrak{a}$ pour $\xi \in \Xi$ et $x=$ $\left(x_{1}, \ldots, x_{r}\right) \in \mathbb{R}^{r}$ par $g_{a}(\xi, x)=\prod_{i=1}^{r}\left(\frac{\sin \left(a x_{i}\right)}{a x_{i}}\right)^{2 N}$.

D'après le lemme 2.4 de [3], pour tout $a \in \mathbb{R}$, on a $g_{a} \in \mathcal{H}_{\Xi}$. De plus, la fonction $\Phi$ étant à support compact, nous pouvons fixer $a, b \in \mathbb{R}^{+}$tels que, pour tout $(\xi, x) \in \Xi \times \mathbb{R}^{r}$, on ait

$$
|\Phi(\xi, x)| \leq b g_{a}(x)
$$

Ce qui est suffisant.

10.2.0.7. Perturbation des opérateurs de Fourier. — Sous l'hypothèse R3, la fonction

$$
\mathbb{R}^{r} \rightarrow \operatorname{End}(L(\Xi)) \quad \lambda \mapsto Q_{\lambda}
$$

est de classe $C^{\infty}$ sur $\mathbb{R}^{r}$. Ainsi, par la théorie des perturbations, les propriétés spectrales de $Q_{\lambda}$ sont essentiellement les mêmes que celles de $Q_{0}$ lorsque $\lambda$ est proche de 0 . Plus précisément, il existe un réel $\delta>0$ tel que, pour tout $\lambda \in \mathbb{B}_{\mathbb{R}^{r}}(0, \delta)$, on ait

$$
Q_{\lambda}=k(\lambda) p_{\lambda}+R_{\lambda}
$$

où

- $k_{\lambda}$ est la valeur spectrale de plus haut module de l'opérateur de Fourier $Q_{\lambda}$; notons que $k_{\lambda}$ est une valeur propre et qu'elle est isolée dans le spectre de $Q_{\lambda}$. 
- $p_{\lambda}$ est le projecteur spectral associé à $k(\lambda)$; c'est un projecteur de rang 1 .

- $R_{\lambda}$ est un endomorphisme de $L(\Xi)$ de rayon strictement inférieur à 1 tel que $p_{\lambda} \circ R_{\lambda}=R_{\lambda} \circ p_{\lambda}=0$.

D'après l'hypothèse $\mathrm{R} 1$, on a $k(0)=1$ et, pour tout $\phi \in L(\Xi)$, on a

$$
p_{0}(\phi)=1_{\Xi} \int_{\Xi} \phi d \nu \text { et } R_{0}(\phi)=R(\phi) .
$$

L'intérêt de la décomposition (4) réside dans le fait qu'elle permet une écriture explicite de la singularité en 0 de la résolvante

$$
\left(I-Q_{\lambda}\right)^{-1} \text {. }
$$

Soulignons que cette résolvante est bien définie, pour $\lambda$ non nul, car la probabilité de transition $\tilde{Q}$ vérifie l'hypothèse $\mathrm{R} 2$; en effet, pour tout $\lambda \neq 0$ et tout entier $n \in \mathbb{N}$, on a

$$
Q_{\lambda}^{n}=k(\lambda)^{n} p_{\lambda}+R_{\lambda}^{n}
$$

si bien que

$$
\left(I-Q_{\lambda}\right)^{-1}=\frac{p_{\lambda}}{1-k(\lambda)}+\left(I-R_{\lambda}\right)^{-1} .
$$

Le lemme qui suit précise le développement en 0 de la fonction $\lambda \mapsto k(\lambda)$.

Lemme 10.6. - (Proposition 1.22 de [2]) Sous les hypothèses R0, R1, R2, R3 et $\mathrm{R} 4$. Il existe une matrice $S$ carrée d'ordre $r$, symétrique et définie positive telle que, pour tout $\lambda \in \mathbb{R}^{r}$, on ait

$$
k(\lambda)=1+i\langle\vec{M}, \lambda\rangle-\frac{1}{2}\langle S(\lambda), S(\lambda)\rangle+o\left(\|\lambda\|^{2}\right)
$$

10.2.0.8. Ecriture du noyau potentiel sous la forme d'une intégrale singulière. - Pour tout $\lambda \in \mathbb{R}^{r} \backslash\{0\}$, on note $k(\lambda) \in \mathbb{R}, p_{\lambda} \in \operatorname{End}(L(\Xi))$ et $R_{\lambda} \in$ End $(L(\Xi))$ comme dans la section précédente.

Lemme 10.7. - (Deuxième partie, section D de [2]) Avec les notations introduites ci-dessus. Si $\kappa$ est une fonction $C^{\infty}$ à support compact sur $\mathbb{R}^{r}$ valant 1 sur $\mathbb{B}_{\mathbb{R}^{r}}(0, \delta)$ alors, pour toute fonction $\phi$ lipschitzienne sur $\Xi$ et toute fonction $u$ continue sur $\mathbb{R}^{r}$ dont la transformée de Fourier $\hat{u}$ est intégrable, on a :

$$
\begin{aligned}
\mathbb{E}_{(\xi, \alpha)}\left(\sum_{n=0}^{+\infty} \phi \otimes u\left(X_{n}\right)\right) & =\frac{1}{(2 \pi)^{r}} \int_{\mathbb{R}^{r}} e^{i\langle\lambda, \alpha\rangle} \kappa(\lambda) \frac{p_{\lambda} \phi(\xi)}{1-k(\lambda)} \hat{u}(-\lambda) d \lambda \\
& +\frac{1}{(2 \pi)^{r}} \int_{\mathbb{R}^{r}} e^{i\langle\lambda, \alpha\rangle} \kappa(\lambda)\left(I-R_{\lambda}\right)^{-1} \phi(\xi) \hat{u}(-\lambda) d \lambda \\
& +\frac{1}{(2 \pi)^{r}} \int_{\mathbb{R}^{r}} e^{i\langle\lambda, \alpha\rangle}(1-\kappa(\lambda))\left(I-Q_{\lambda}\right)^{-1} \phi(\xi) \hat{u}(-\lambda) d \lambda .
\end{aligned}
$$

TOME $137-2009-\mathrm{N}^{\circ} 3$ 
10.2.0.9. Démonstration du théorème 10.2. - D'après les corollaires 10.4 et 10.5 , il suffit de montrer que, pour tout $\phi \in L(\Xi)$ et tout $u \in \mathcal{H}$, la fonction

$$
\mathfrak{a} \rightarrow \mathbb{R} \quad \alpha \mapsto\langle\vec{M}, \alpha\rangle^{\frac{r-1}{2}} \sum_{n=0}^{+\infty} \mathbb{E}_{(\xi, \alpha)}\left(\phi \otimes u\left(X_{n}\right)\right)
$$

est bornée et que

$$
\begin{aligned}
\lim _{t \rightarrow+\infty} \sup _{\xi \in \Xi} \mid t^{\frac{r-1}{2}} \mathbb{E}_{(\xi,-t \vec{M}-\sqrt{t} f)} \sum_{n=0}^{+\infty}\left(\phi \otimes u\left(X_{n}\right)\right) \\
-c e^{-\frac{\|S(f)\|^{2}\|S(\vec{M})\|^{2}-\langle S(f), S(\vec{M})\rangle^{2}}{2\|S(\vec{M})\|^{2}}} \int_{\Xi \times \mathbb{R}^{r}} \phi \otimes u(\xi, \alpha) d \nu(\xi) \otimes d \alpha \mid=0 .
\end{aligned}
$$

D'après le lemme 10.7 , on a

$$
\begin{aligned}
\sum_{n=0}^{+\infty} \mathbb{E}_{(\xi, \alpha)}\left(\phi \otimes u\left(X_{n}\right)\right) & =\frac{1}{(2 \pi)^{r}} \int_{\mathbb{R}^{r}} e^{i\langle\lambda, \alpha\rangle} \kappa(\lambda) \frac{p_{\lambda} \phi(\xi)}{1-k(\lambda)} \hat{u}(-\lambda) d \lambda \\
& +\frac{1}{(2 \pi)^{r}} \int_{\mathbb{R}^{r}} e^{i\langle\lambda, \alpha\rangle} \kappa(\lambda)\left(I-R_{\lambda}\right)^{-1} \phi(\xi) \hat{u}(-\lambda) d \lambda \\
& +\frac{1}{(2 \pi)^{r}} \int_{\mathbb{R}^{r}} e^{i\langle\lambda, \alpha\rangle}(1-\kappa(\lambda))\left(I-Q_{\lambda}\right)^{-1} \phi(\xi) \hat{u}(-\lambda) d \lambda .
\end{aligned}
$$

Les fonctions $\mathbb{R}^{r} \rightarrow \mathbb{R}, \lambda \mapsto\left(I-R_{\lambda}\right)^{-1} \phi(\xi) \hat{u}(-\lambda)$ et $\mathbb{R}^{r} \rightarrow \mathbb{R}, \lambda \mapsto$ $(1-\kappa(\lambda))\left(I-Q_{\lambda}\right)^{-1} \phi(\xi) \hat{u}(-\lambda)$ étant $C^{N}$ à support compact sur $\mathbb{R}^{r}$, avec $N \geq r$, leurs dérivées successives sont bornées dans $L^{1}\left(\mathbb{R}^{r}, d \lambda\right)$ uniformément par rapport à $\xi$; en effectuant alors plusieurs intégrations par parties, on montre que, pour tout $n \in\{0, \ldots, N\}$, on a :

$$
\sup _{\xi \in \Xi}\|\alpha\|^{n} \int_{\mathbb{R}^{r}} e^{i\langle\lambda, \alpha\rangle} \kappa(\lambda)\left(I-R_{\lambda}\right)^{-1} \phi(\xi) \hat{u}(\lambda) d \lambda \rightarrow 0
$$

et

$$
\sup _{\xi \in \Xi}\|\alpha\|^{n} \int_{\mathbb{R}^{r}} e^{i\langle\lambda, \alpha\rangle}(1-\kappa(\lambda))\left(I-Q_{\lambda}\right)^{-1} \phi(\xi) \hat{u}(\lambda) d \lambda \rightarrow 0
$$

lorsque $\|\alpha\| \rightarrow+\infty$. Il nous reste à estimer le comportement lorsque $t \rightarrow \infty$ de la fonction

$$
t \mapsto \int_{\mathbb{R}^{r}} e^{-i\langle\lambda, t \vec{M}+\sqrt{t} f\rangle} \kappa(\lambda) \frac{p_{\lambda} \phi(\xi)}{1-k(\lambda)} \hat{u}(-\lambda) d \lambda .
$$

Pour ce faire, nous utilisons le 
LEMme 10.8. - (2.42 + 2.43 de [2] ) Avec les notations introduites ci-dessus. Soit $F$ la fonction définie sur $\Xi \times \mathbb{R}^{r}$ par

$$
\begin{aligned}
F(\xi, \alpha)=\int_{\mathbb{R}^{r}} e^{i\langle\lambda, \alpha\rangle} \kappa(\lambda) & \frac{p_{\lambda} \phi(\xi)}{1-k(\lambda)} \hat{u}(-\lambda) d \lambda \\
& \quad-C(\xi) \int_{\mathbb{R}^{r}} e^{i\langle\lambda, \alpha\rangle} \frac{\kappa(\lambda)}{-i\langle\vec{M}, \lambda\rangle+\frac{1}{2}\langle S(\lambda), S(\lambda)\rangle} d \lambda .
\end{aligned}
$$

On a

$$
\sup _{\xi \in \Xi} F(\xi, \alpha)=o\left(\|\alpha\|^{\frac{1-r}{2}}\right)
$$

et il nous suffit de préciser le comportement en $+\infty$ de :

$$
t \mapsto t^{\frac{r-1}{2}} \int_{\mathbb{R}^{r}} e^{-i\langle\lambda, t \vec{M}+\sqrt{t} f\rangle} \frac{\hat{u}(-\lambda)}{-i\langle\vec{M}, \lambda\rangle+\frac{1}{2}\langle S(\lambda), S(\lambda)\rangle} d \lambda .
$$

Nous avons le

LEMme 10.9. - Soient $\vec{m}$ un vecteur de $\mathbb{R}^{r}$ et $S_{1}$ une matrice carrée d'ordre $r$, symétrique et définie positive. Il existe une constante $c>0$ telle que, pour tout vecteur $f$ et toute fonction $v$ continue sur $\mathbb{R}^{r}$ et dont la transformée de Fourier $\hat{v}$ est à support compact, on ait :

$$
\begin{aligned}
& t^{\frac{r-1}{2}} \int_{\mathbb{R}^{r}} e^{-i\langle\lambda, t \vec{m}+\sqrt{t} f\rangle} \frac{\hat{v}(-\lambda)}{-i\langle\vec{m}, \lambda\rangle+\frac{1}{2}\left\langle S_{1}(\lambda), S_{1}(\lambda)\right\rangle} d \lambda \\
& \quad \rightarrow c e^{-\frac{\left\|S_{1}(f)\right\|^{2}\left\|S_{1}(\vec{m})\right\|^{2}-\left\langle S_{1}(f), S_{1}(\vec{m})\right\rangle^{2}}{2\left\|S_{1}(\vec{m})\right\|^{2}}} \int_{\mathbb{R}^{r}} v(\alpha) d \alpha
\end{aligned}
$$

lorsque $t \rightarrow+\infty$.

On en déduit

$$
\begin{aligned}
\int_{\mathbb{R}^{r}} & e^{-i\langle\lambda, t \vec{M}+\sqrt{t} f\rangle} \kappa(\lambda) \frac{p_{\lambda} \phi(\xi)}{1-k(\lambda)} \hat{u}(-\lambda) d \lambda \\
& \rightarrow c e^{-\frac{\|S(f)\|^{2}\|S(\vec{M})\|^{2}-\langle S(f), S(\vec{M})\rangle^{2}}{2\|S(\vec{M})\|^{2}}} \int_{\Xi} \phi(\xi) d \nu(\xi) \int_{\mathbb{R}^{r}} u(\alpha) d \alpha
\end{aligned}
$$

lorsque $t \rightarrow+\infty$. Ce qui est suffisant d'après ce qui précède.

\section{BIBLIOGRAPHIE}

[1] P. Albuquerque - «Uniqueness of conformal densities and the semiflow of Weyl chambers », C. R. Acad. Sci. Paris Sér. I Math. 326 (1998), p. $1105-1110$. 
[2] M. Babillot - «Théorie du renouvellement pour des chaînes semimarkoviennes transientes », Ann. Inst. H. Poincaré Probab. Statist. 24 (1988), p. 507-569.

[3] M. BABILlot \& F. LedRAPPIER - «Lalley's theorem on periodic orbits of hyperbolic flows », Ergodic Theory Dynam. Systems 18 (1998), p. 17-39.

[4] M. Babillot \& M. Peigné - «Homologie des géodésiques fermées sur des variétés hyperboliques avec bouts cuspidaux», Ann. Sci. École Norm. Sup. 33 (2000), p. 81-120.

[5] Y. Benoist - «Propriétés asymptotiques des groupes linéaires », Geom. Funct. Anal. 7 (1997), p. 1-47.

[6] _ « Propriétés asymptotiques des groupes linéaires. II », in Analysis on homogeneous spaces and representation theory of Lie groups, OkayamaKyoto (1997), Adv. Stud. Pure Math., vol. 26, Math. Soc. Japan, 2000, p. $33-48$.

[7] F. DAL'Bo \& M. Peigné - «Groupes du ping-pong et géodésiques fermées en courbure -1», Ann. Inst. Fourier (Grenoble) 46 (1996), p. 755-799.

[8] _ _ « Some negatively curved manifolds with cusps, mixing and counting », J. reine angew. Math. 497 (1998), p. 141-169.

[9] Y. GuivarC'H - « Produits de matrices aléatoires et applications aux propriétés géométriques des sous-groupes du groupe linéaire », Ergodic Theory Dynam. Systems 10 (1990), p. 483-512.

[10] Y. GuivarC'H \& J. HARDY - «Théorèmes limites pour une classe de chaînes de Markov et applications aux difféomorphismes d'Anosov », Ann. Inst. H. Poincaré Probab. Statist. 24 (1988), p. 73-98.

[11] J.-F. QUiNT - «Divergence exponentielle des sous-groupes discrets en rang supérieur », Comment. Math. Helv. 77 (2002), p. 563-608.

[12] _ « Mesures de Patterson-Sullivan en rang supérieur », Geom. Funct. Anal. 12 (2002), p. 776-809.

[13] _ « Groupes de Schottky et comptage », Ann. Inst. Fourier (Grenoble) 55 (2005), p. 373-429.

[14] T. Roblin - «Ergodicité et équidistribution en courbure négative », Mém. Soc. Math. Fr. (N.S.) 95 (2003).

[15] X. Thirion - « Mesures de Patterson des groupes de Ping-Pong » (soumis). 